\title{
Intrathecal bone marrow stromal cells inhibit neuropathic pain via TGF- $\beta$ secretion
}

\author{
Gang Chen, ${ }^{1,2}$ Chul-Kyu Park, ${ }^{1,3}$ Rou-Gang Xie, ${ }^{1,4,5}$ and Ru-Rong ji1,6 \\ 'Department of Anesthesiology, Duke University Medical Center, Durham, North Carolina, USA. ${ }^{2}$ Jiangsu Key Laboratory of Neuroregeneration, Co-Innovation Center of Neuroregeneration, \\ Nantong University, Nantong, China. ${ }^{3}$ Department of Physiology, College of Medicine, Gachon University, Incheon, South Korea. ${ }^{4}$ Department of Anesthesiology and Pain Management, Xijing Hospital, and \\ ${ }^{5}$ Institute of Neuroscience, Fourth Military Medical University, Xian, China. ${ }^{6}$ Department of Neurobiology, Duke University Medical Center, Durham, North Carolina, USA.
}

\begin{abstract}
Neuropathic pain remains a pressing clinical problem. Here, we demonstrate that a local, intrathecal (i.t.) injection of bone marrow stromal cells (BMSCs) following lumbar puncture alleviates early- and late-phase neuropathic pain symptoms, such as allodynia and hyperalgesia, for several weeks in murine chronic constriction injury (CCI) and spared nerve injury models. Moreover, i.t. BMSCs reduced CCl-induced spontaneous pain and axonal injury of dorsal root ganglion (DRC) neurons and inhibited CCl-evoked neuroinflammation in DRGs and spinal cord tissues. BMSCs secreted TCF- $\beta 1$ into the cerebrospinal fluid, and neutralization of TGF- $\beta 1$, but not IL-10, reversed the analgesic effect of BMSCs. Conversely, i.t. administration of TCF- $\beta 1$ potently inhibited neuropathic pain. TCF- $\beta 1$ acted as a powerful neuromodulator and rapidly (within minutes) suppressed CCIevoked spinal synaptic plasticity and DRG neuronal hyperexcitability via TGF- $\beta$ receptor 1 -mediated noncanonical signaling. Finally, nerve injury upregulated CXCL12 in lumbar L4-L6 DRGs, and this upregulation caused migration of i.t.-injected BMSCs to DRGs through the CXCL12 receptor CXCR4, which was expressed on BMSCs. BMSCs that migrated from the injection site survived at the border of DRGs for more than 2 months. Our findings support a paracrine mechanism by which i.t. BMSCs target CXCL12-producing DRGs to elicit neuroprotection and sustained neuropathic pain relief via TCF- $\beta 1$ secretion.
\end{abstract}

\section{Introduction}

Treatment of neuropathic pain triggered by multiple insults to the nervous system is a clinical challenge, because the pathogenesis of this hypersensitive state is very complex, involving structural and neurophysiological changes throughout the neuroaxis, from the site of the peripheral nerve injury, to the cell bodies of primary sensory neurons in dorsal root ganglion (DRG) neurons, and to the spinal cord and brain (1). Currently, there are no drugs that can treat neuropathic pain in a complete and definitive way $(2,3)$.

Bone marrow stromal cells (BMSCs) are a population of progenitor cells of mesodermal origin that are present in the BM of adults and give rise to various tissues throughout the body (4). BMSCs have emerged as a major source for cell-based therapies, with a diverse spectrum of potential clinical applications. Because of their high expansion potential, genetic stability, stable phenotype, and strong immunosuppressive properties, BMSCs can be exploited for successful autologous and heterologous transplantation, without requiring immune suppressants (5). In addition, BMSCs can be easily collected and shipped from the laboratory to the bedside and are compatible with different delivery methods and formulations. BMSCs were originally con-

Note regarding evaluation of this manuscript: Manuscripts authored by scientists associated with Duke University, The University of North Carolina at Chapel Hill, Duke-NUS, and the Sanford-Burnham Medical Research Institute are handled not by members of the editorial board but rather by the science editors, who consult with selected external editors and reviewers.

Conflict of interest: The authors have declared that no conflict of interest exists. Submitted: January 8, 2015; Accepted: May 27, 2015.

Reference information: J Clin Invest. 2015;125(8):3226-3240. doi:10.1172/JCI80883. ceived as stem/progenitor cells to rebuild diseased or damaged tissues. However, systemically infused BMSCs have been shown to exert therapeutic effects through the release of cytokines and trophic factors that act on local, or perhaps distant, target tissues $(6,7)$. Through this paracrine modulation, it has been discovered that BMSCs are potent modulators of immune responses in humans and animals (8).

Systemic or local injection of BMSCs has been shown to alleviate inflammatory and neuropathic pain (refs. 9-14 and also see Supplemental Table 1; supplemental material available online with this article; doi:10.1172/JCI80883DS1). However, several key issues surrounding this potential treatment have not been addressed, thus limiting its clinical applications. First, the molecular mechanisms by which BMSCs attenuate chronic pain are unknown. Second, the destination of the injected or implanted BMSCs is unclear. Third, the analgesic capability of BMSCs, especially that of reversing chronic pain, has not been fully explored. Finally, the optimal conditions for the delivery of BMSCs to treat chronic pain remain to be identified.

In this study, we used an approach involving intrathecal (i.t.) administration of BMSCs to target DRGs and spinal cord in the pain pathway. We found that i.t. BMSCs, given early or late after nerve injury, effectively suppressed neuropathic pain for several weeks in mice after chronic constriction injury (CCI) and spared nerve injury (SNI). Our results also show that BMSCs secreted TGF- $\beta 1$, which acted as a powerful neuromodulator to mediate the antinociceptive effects of BMSCs. Furthermore, our findings demonstrate that transplanted BMSCs selectively targeted injured lumbar DRGs, which produced the chemokine CXCL12 to induce chemotaxis of CXCR4-expressing BMSCs. 

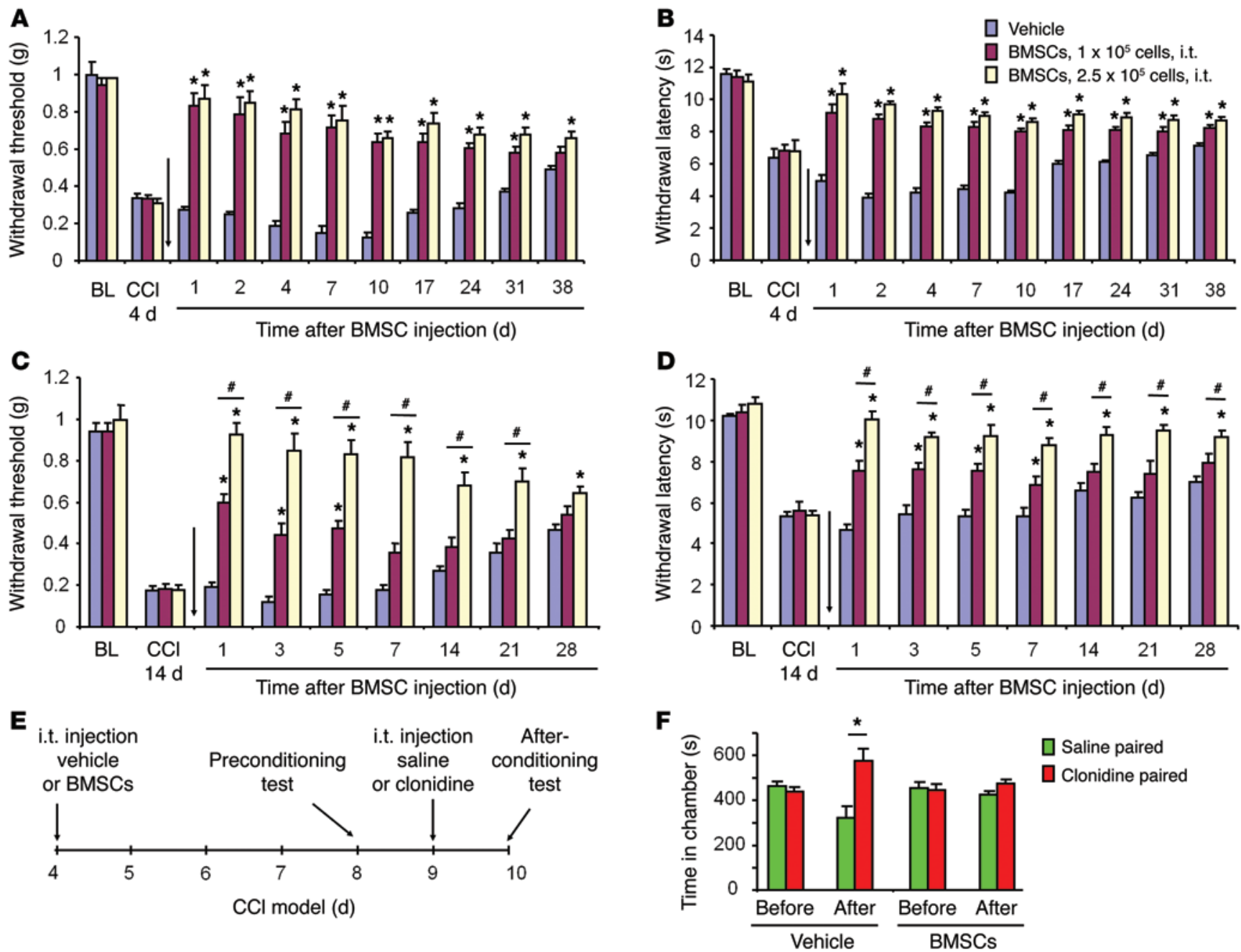

Figure 1. Inhibition of CCl-induced evoked and ongoing neuropathic pain in mice by a single i.t. injection of BMSCs. (A and B) Prolonged inhibition of mechanical allodynia (A) and thermal hyperalgesia (B) for 5 weeks by early treatment with i.t. injection of BMSCs $\left(1.0\right.$ or $2.5 \times 10^{5}$ cells), given 4 days after CCI. ${ }^{*} P<0.05$, compared with vehicle (PBS); $n=6$ mice/group. (C and D) Reversal of mechanical allodynia (C) and thermal hyperalgesia (D) by late treatment with i.t. BMSCs (1.0 or $2.5 \times 10^{5}$ cells), given 14 days after CCI. Arrows in A-D indicate the time of BMSC injection. ${ }^{*} P<0.05$, compared with vehicle; ${ }^{\#} P<0.05 ; n=6$ mice/group. (E) Paradigm for measuring ongoing pain using a 2-chamber CPP test. (F) CCI-induced ongoing pain was abolished by treatment with BMSCs. ${ }^{*} P<0.05$, compared with saline; $n=5$ mice/group. Statistical significance was determined by 2 -way repeated-measures ANOVA, followed by Bonferroni's post-hoc test (A-D) or Student's $t$ test $(\mathbf{F})$. All data are expressed as the mean \pm SEM. BL, baseline.

\section{Results}

Early or late treatment with BMSCs via the i.t. route produces longterm relief of neuropathic pain after CCI. We induced neuropathic pain in mice via CCI of the sciatic nerve (15). In this model, neuropathic pain was characterized by mechanical allodynia and heat hyperalgesia, both of which lasted more than 5 weeks but recovered after 6 to 8 weeks (Figure 1, A and B). To test the hypothesis that BMSCs would alleviate behavioral signs of neuropathic pain, we administered an i.t. injection of BMSCs into spinal cerebrospinal fluid (CSF) via lumbar puncture, 4 days after CCI, when neuropathic pain developed but had not reached its peak. BMSCs (1.0 $\times 10^{5}$ or $2.5 \times 10^{5}$ cells) with a purity of $88 \%$ (Supplemental Figure 1) were prepared in $10 \mu \mathrm{l}$ PBS and collected for i.t. injection. As shown in Figure 1, A and B, a single i.t. injection of BMSCs, given on CCI day 4, produced a rapid (within 1 day) inhibition of CCIinduced mechanical allodynia and thermal hyperalgesia. Notably, these antinociceptive effects lasted for more than 5 weeks follow- ing either low-dose or high-dose BMSC treatment (Figure 1, A and B). We next examined the effects of BMSCs on established latephase neuropathic pain, 14 days after CCI. As shown in Figure 1, $\mathrm{C}$ and D, i.t. BMSCs effectively reversed mechanical allodynia and thermal hyperalgesia in a dose-dependent manner: low doses of BMSCs $\left(1.0 \times 10^{5}\right.$ cells $)$ only reversed mechanical allodynia and heat hyperalgesia for 1 week, whereas high doses of BMSCs $\left(2.5 \times 10^{5}\right.$ cells $)$ reversed these neuropathic pain symptoms for more than 4 weeks (Figure 1, C and D).

Given the limitations of measuring evoked pain and the withdrawal reflex (16), we tested whether BMSCs would also alleviate nerve injury-induced ongoing pain on the basis of the finding that negative reinforcement can unmask ongoing neuropathic pain in a 2-chamber conditioned place preference (CPP) test (17). The paradigm of the CPP tested is described in Figure 1E. The result showed that mice spent more time in the chamber paired with the analgesic clonidine than in the chamber paired with the vehicle 
A

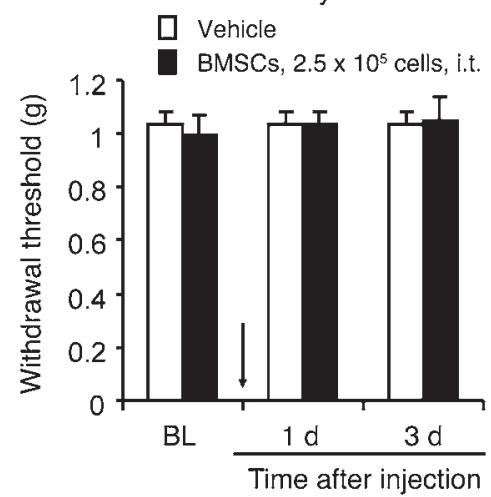

C

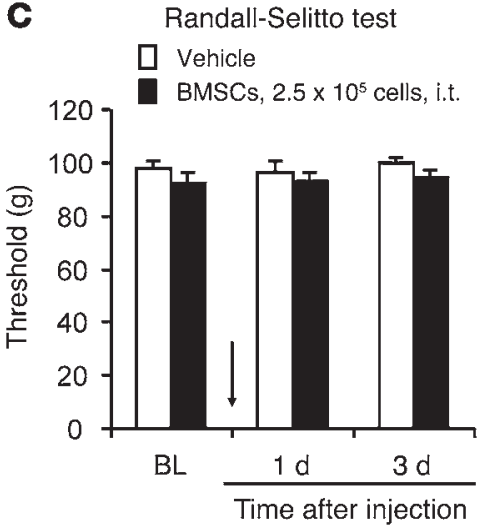

B

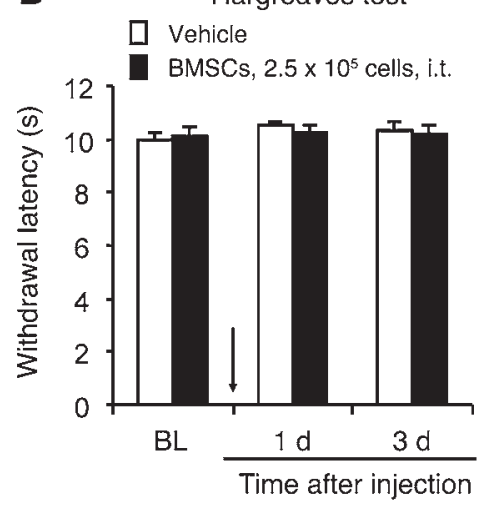

D

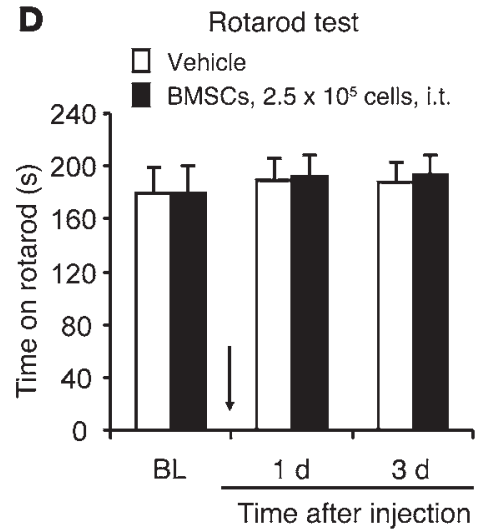

Figure 2. Baseline pain and motor function are not altered by i.t. injection of BMSCs. (A-C) Baseline pain sensitivity evaluated by the von Frey test for mechanical sensitivity (A), the Hargreaves test for heat sensitivity (B), and the Randall-Selitto test for mechanical sensitivity. (D) Rotarod test for the evaluation of motor function. $n=6$ mice/group. Arrows in $\mathbf{A}-\mathbf{D}$ indicate the time of BMSC injection.

injection (Figure $1 \mathrm{~F}$ ), indicating that the mice developed ongoing pain after CCI. Importantly, CCI-induced ongoing pain was abolished by i.t. treatment with BMSCs $\left(2.5 \times 10^{5}\right.$ cells, Figure $\left.1 \mathrm{~F}\right)$.

While chronic pain is destructive, physiological pain is protective. We also tested whether i.t. BMSCs would affect physiological pain (basal pain) under normal conditions. Notably, i.t. BMSC treatment, even at a high dose $\left(2.5 \times 10^{5}\right.$ cells $)$, did not change the mechanical or thermal pain thresholds in naive mice (Figure 2, A-C), nor did this treatment affect motor function, as evaluated by a rotarod test (Figure 2D). Collectively, these data suggest that i.t. injection of BMSCs, either in the early or late phase of CCI, can elicit long-lasting relief of neuropathic pain symptoms, without affecting baseline pain thresholds (physiological pain states) or motor function.

BMSCs delivered i.t. protect primary sensory neurons from CCIinduced axonal injury. The activating transcription factor 3 (ATF3) is a widely used marker for DRG neuron injury (18). ATF3 immunoreactivity (IR) was barely detected in DRG neurons in sham surgery mice, but had markedly increased to $40 \%$ of the neurons in lumbar L4-L5 DRGs after CCI (Figure 3, A-C). Treatment with i.t. BMSCs $\left(2.5 \times 10^{5}\right.$ cells $)$, given 4 days after CCI, inhibited CCIinduced ATF3 expression in DRG neurons 8 days after CCI, and the percentage of ATF3 IR neurons was reduced to $14 \%$ after BMSC treatment (Figure 3, A-C). Nerve injury is also known to downregulate the neuropeptide calcitonin gene-related peptide (CGRP) in peptidergic neurons as well as isolectin B4 (IB4) binding in nonpeptidergic neurons in DRGs (19, 20). Notably, i.t. BMSCs reversed CCI-induced downregulation of both CGRP and IB4 staining in DRG neurons (Figure 3, D and E) and also in their central axonal terminals in the spinal cord dorsal horn (Figure 3, F and G). These results suggest that BMSCs administered i.t. can protect DRG neurons from axonal injury.

BMSCs delivered i.t. control neuroinflammation in DRGs and spinal cord after CCI. Neuroinflammation is an inflammatory response in the PNS and CNS and has been strongly implicated in the genesis of neuropathic pain $(21,22)$. CCI caused a marked increase in IBA-1-labeled macrophages in injured L4-L5 DRGs, but this increase on CCI day 8 was blocked by i.t. BMSC treatment $\left(2.5 \times 10^{5}\right.$ cells $)$ given on CCI day 4 (Figure 4, A-C). Quantitative PCR (qPCR) revealed that CCI also induced upregulation of Gfap (a marker for satellite glial cells) and $I l 1 b$ and Il6, but not Tnf (key proinflammatory cytokines), in the L4-L5 DRGs, and the upregulation of all 4 of these mRNAs was suppressed by treatment with BMSCs (Figure 4D).

Neuroinflammation is also characterized by the activation of glial cells, including microglia and astroctyes in spinal cord, and this activation plays an essential role in the pathogenesis of neuropathic pain by producing Il-1 $\beta$, IL-6, and TNF to enhance spinal cord synaptic transmission (23-27). Eight days after surgery, CCI caused marked increases in the IR of GFAP (astrocyte marker) and IBA-1 (microglial marker), as well as morphological changes in astrocytes and microglia on the ipsilateral side of the spinal dorsal horn. All of these glial changes were attenuated by treatment with BMSCs (Figure 4, E and F). qPCR results showed that CCI induced the upregulation of Iba1, Il1b, Il6, and Tnf transcripts in the L4-L5 spinal dorsal horn, all of which were suppressed by BMSCs (Figure $4 G)$. Thus, i.t. BMSCs can effectively control neuroinflammation in the neuroaxis of pain, which includes DRGs and spinal cord.

BMSCs release TGF- $\beta 1$ to inhibit neuropathic pain. Given the rapid analgesic effect observed on day 1 , we reasoned that BMSCs might control neuropathic pain by paracrine signaling through the secretion of potential "pain killers." The antiinflammatory cytokines TGF- $\beta$ and IL-10 have been implicated in the beneficial effects of BMSCs in asthma and sepsis $(28,29)$. TGF- $\beta$ and IL-10 also inhibit neuropathic pain and spinal neuroinflammation after nerve injury $(30,31)$. To assess their involvement in BMSC-elicited pain relief, we measured TGF- $\beta 1$ and IL-10 release in BMSC culture medium. We detected high basal release of TGF- $\beta 1(120 \mathrm{pg} / \mathrm{ml})$, but very low basal release of IL-10 $(<5 \mathrm{pg} /$ $\mathrm{ml}$ ) (Figure 5A). We next examined whether inflammatory challenge would boost the release of IL-10 and TGF- $\beta 1$. Exposure of BMSCs to TNF- $\alpha$ or LPS significantly increased the release of TGF- $\beta 1$ but not IL-10 (Figure $5 \mathrm{~A}$ ). Furthermore, we examined TGF- $\beta 1$ release in CSF collected from naive and CCI mice 
A
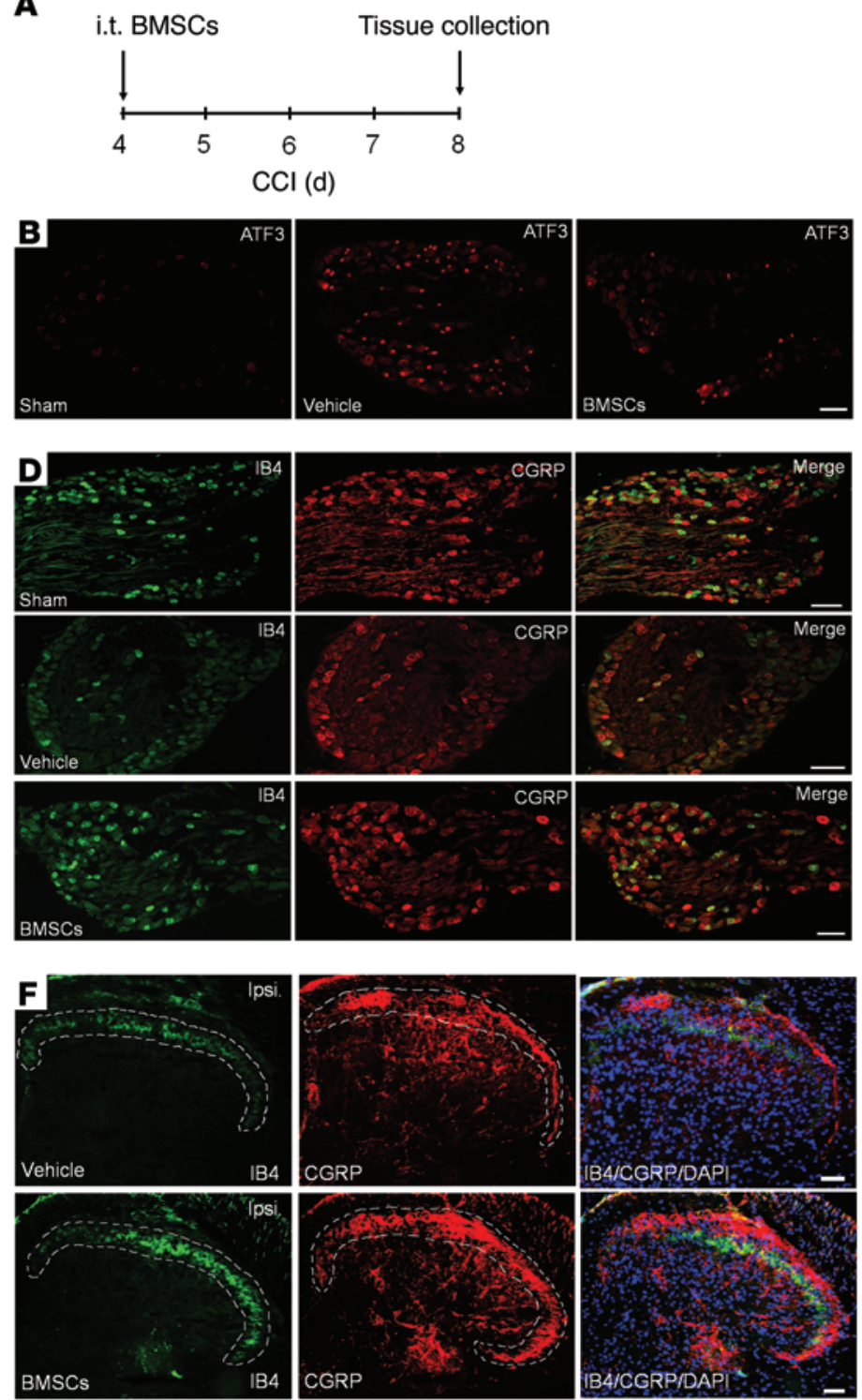

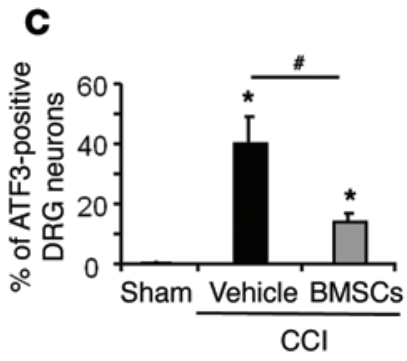

\section{$\mathbf{E}$ \\ Sham \\ $\mathrm{CCl}$, vehicle \\ $\mathrm{CCCl}, \mathrm{BMSCs}$}
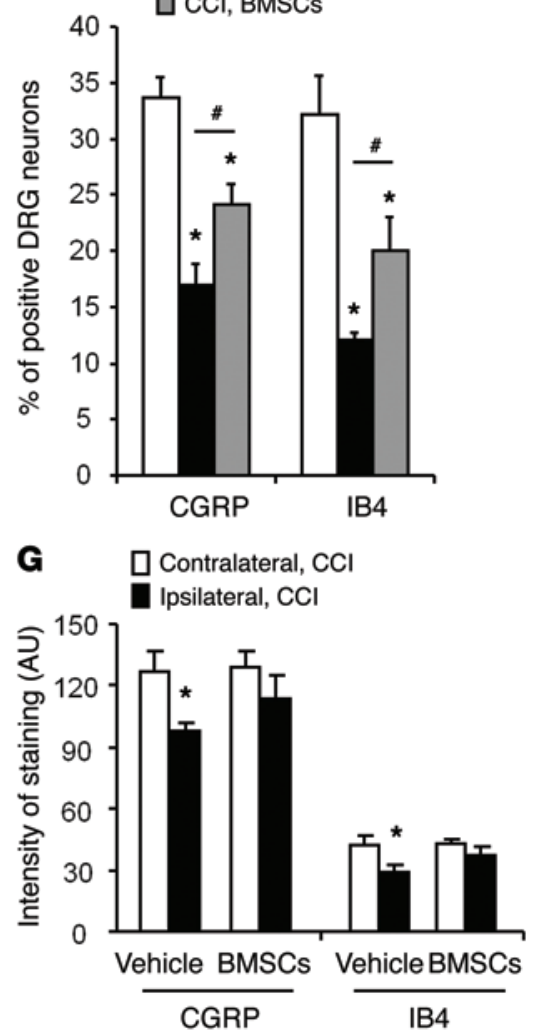

Figure 3. BMSCs administered i.t. inhibit CCI-induced upregulation of ATF3 in DRGs and reduce CCI-induced downregulation of CGRP and IB4 in DRGs and the spinal cord dorsal horn. (A) Paradigm showing the timing of BMSC treatment (CCI day 4) and tissue collection (CCI day 8). (B-G) Inhibition of CCI-induced upregulation of ATF3 (B and $\mathbf{C}$ ) and downregulation of IB4 and CGRP ( $\mathbf{D}$ and $\mathbf{E}$ ) in L4-L5 DRGs, as well as downregulation of IB4 and CGRP in the L4-L5 spinal cord dorsal horn (F and $\mathbf{C})$ by i.t. injection of BMSCs $\left(2.5 \times 10^{5}\right.$ cells, 4 days after CCI). Scale bars: $50 \mu \mathrm{m}$ (B, D, and F). Quantification results of ATF3 staining in DRGs (C), IB4 and CGRP staining in DRGs (E), and IB4 and CGRP staining in dorsal horns (G). ${ }^{*} P<0.05$, compared with the sham or contralateral group; ${ }^{~} P<0.05 ; n=4-5$ mice/group. Statistical significance was determined by 1-way ANOVA, followed by Bonferroni's post-hoc test. All data are expressed as the mean \pm SEM.

with or without BMSC treatment. TGF- $\beta 1$ release in CSF did not increase in CCI mice, but was substantially increased after they received an i.t. injection of BMSCs (Figure 5B).

To determine whether TGF- $\beta 1$ and IL-10 contribute to the antinociceptive effects of BMSCs in neuropathic pain, we treated mice with a specific neutralizing Ab against TGF- $\beta$ or IL-10, 3 days after injection of BMSCs (i.e., 7 days after CCI). Strikingly, the antiallodynic effect of BMSCs was reversed by i.t. TGF- $\beta$ neutralization, but not by control serum and IL-10 neutralization (Figure $5 \mathrm{C})$. The reversal induced by TGF- $\beta$ neutralization was transient and recovered after 24 hours (Figure 5 C). We further tested TGF- $\beta$ neutralization at 2 additional time points: an earlier time point
(1 day after BMSC injection) and a later time point (24 days after BMSC injection). Notably, TGF- $\beta$ Ab was still capable of reversing BMSC-induced inhibition of allodynia and hyperalgesia at 1 or 24 days after BMSC injection (Supplemental Figure 2, A-D). Twenty-eight days after BMSC injection, TGF- $\beta 1$ levels in L5 DRGs, but not in CSF, were still elevated compared with the levels detected in the vehicle control (Supplemental Figure 2, E and F).

To further validate the critical role of TGF- $\beta 1$ release in neuropathic pain relief with BMSC treatment, we knocked down TGF- $\beta 1$ expression in BMSCs by treating BMSC cultures with a specific siRNA $(1 \mu \mathrm{g} / \mathrm{ml})$ for 18 hours. This treatment resulted in a $67 \%$ reduction in TGF- $\beta 1$ release and a $55 \%$ reduction in TGF- $\beta 1$ expres- 


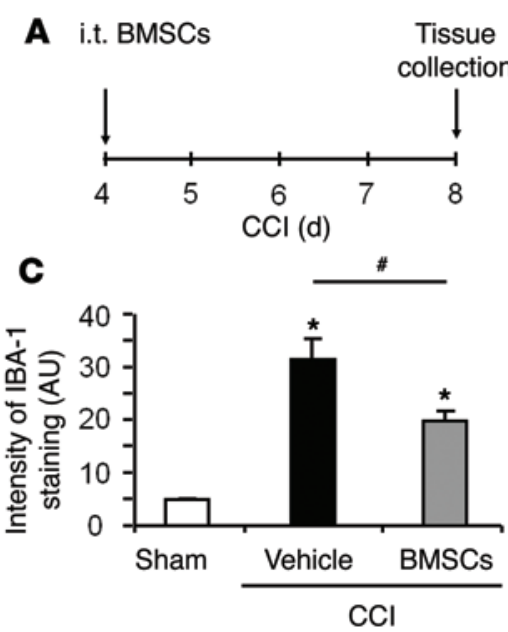

$\mathbf{E}$
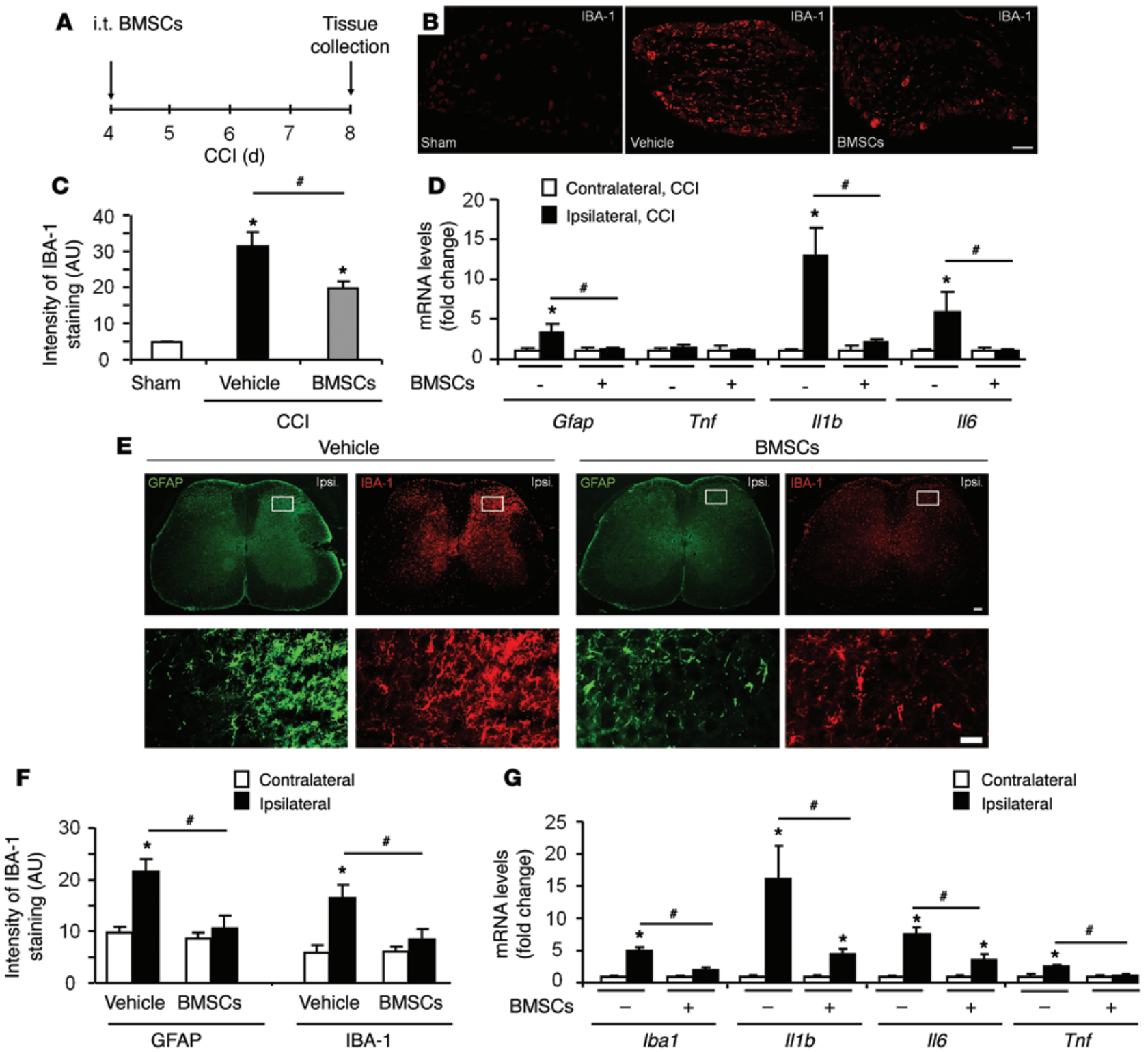

Figure 4. BMSCs administered i.t. inhibit CCI-induced glial activation and neuroinflammation in lumbar DRGs and the spinal cord dorsal horn. (A) Paradigm showing the timing of BMSC treatment (CCI day 4) and tissue collection (CCI day 8). (B) Inhibition of CCI-induced upregulation of the macrophage marker IBA-1 in L4-L5 DRGs by i.t. injection of BMSCs $\left(2.5 \times 10^{5}\right.$ cells). Scale bar: $50 \mu \mathrm{m}$. (C) Quantification of IBA- 1 staining. ${ }^{*} P<0.05$, compared with the sham group; ${ }^{*} P<0.05$; $n=4$ mice/group. (D) qPCR showing expression levels of Gfap, II1b, II6, and Tnf mRNAs in L4-L5 DRGs and the effects of BMSCs. ${ }^{*} P<0.05$, compared with the contralateral group; ${ }^{~} P<0.05 ; n=4$ mice/group. (E and $\mathbf{F}$ ) BMSC inhibition of CCI-induced upregulation of the microglial marker IBA- 1 and the astrocyte marker GFAP in the L4-L5 dorsal horn. Graph in $\mathbf{F}$ shows the quantification of GFAP and IBA-1 staining. Scale bar: $200 \mu \mathrm{m}$ (top panels) and $50 \mu \mathrm{m}$ (bottom panels). Bottom panels are enlarged images of the top panels. ${ }^{*} P<0.05$, compared with the contralateral group; ${ }^{\#} P<0.05 ; n=4$ mice/group. (C) qPCR showing the expression levels of $I b a 1, \| 1 b$, II6, and $T n f$ mRNAs and the effects of BMSCs. ${ }^{*} P<0.05$, compared with the contralateral group; ${ }^{\#} P<0.05 ; n=4-5$ mice/group. Statistical significance was determined by 1-way ANOVA, followed by Bonferroni's post-hoc test. All data are expressed as the mean \pm SEM.

sion in BMSC cultures compared with nontargeting siRNA treatment (Figure 5D). The BMSC-induced antiallodynic effect was compromised for several days after i.t. administration of siRNAtreated BMSCs (Figure 5E and Supplemental Figure 3).

Since only $88 \%$ of cells in the BM cultures expressed the stem cell marker CD90 (Supplemental Figure 1C), we next assessed whether the remaining CD90-negaive cells would also modulate pain via TGF- $\beta 1$. After cell sorting, we amplified CD90-negative cells and collected them to test their analgesic effects. Delivery of these CD90-negative cells by i.t. injection 2 weeks after CCI elicited only mild or transient inhibition of CCI-induced allodynia and hyperalgesia (Supplemental Figure 4, A and B). Furthermore, these CD90-negative cells secreted much less TGF- $\beta 1$ than did CD90-positive cells (Supplemental Figure 4C). Together, these results strongly suggest that i.t. BMSCs inhibit neuropathic pain symptoms via TGF- $\beta 1$ release.

Exogenous TGF- $\beta 1$ attenuates neuropathic pain through TGF- $\beta R 1$. To further test the hypothesis that TGF- $\beta 1$ is sufficient to alleviate behavioral signs of neuropathic pain, we evaluated the antinociceptive effects of exogenous recombinant TGF- $\beta 1$ on CCI-induced 
A
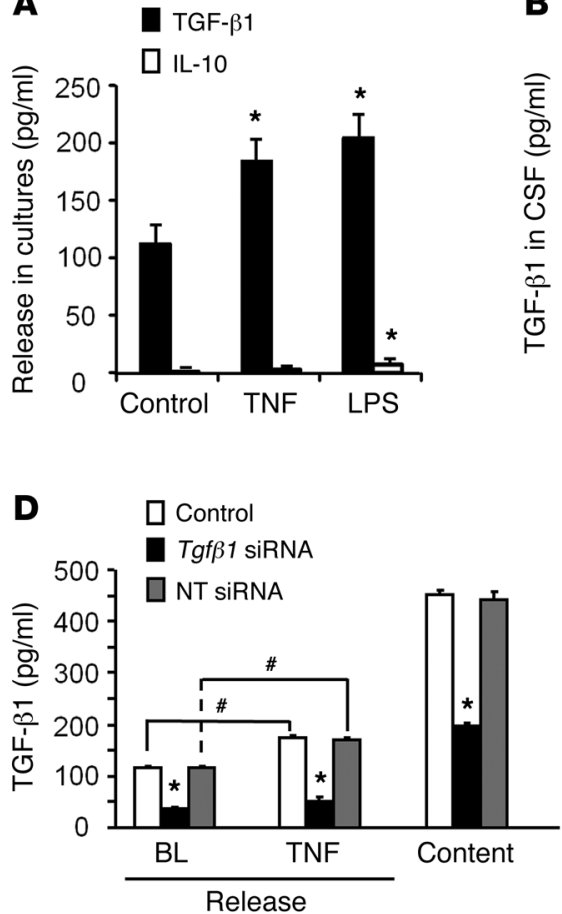

F $\quad \square$ vehicle

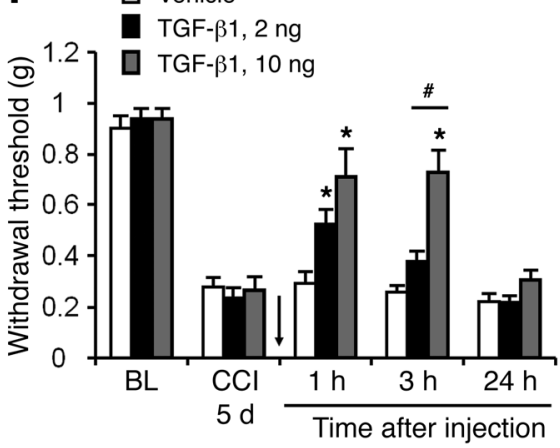

B

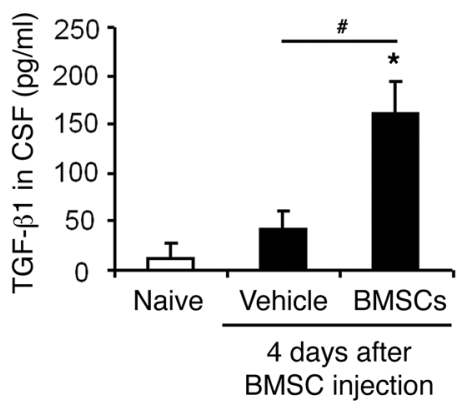

E $\quad \mathrm{NT}$ siRNA-treated BMSCs, i.t.

을 1.2 Tgf $\beta 1$ siRNA-treated BMSCs, i.t.

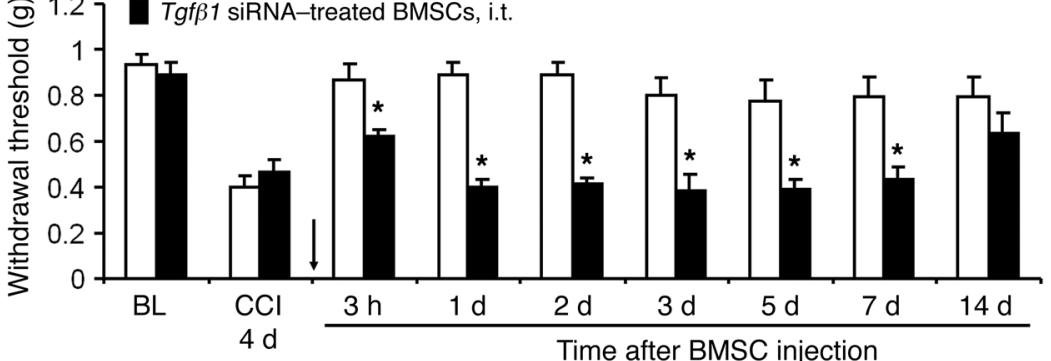

G

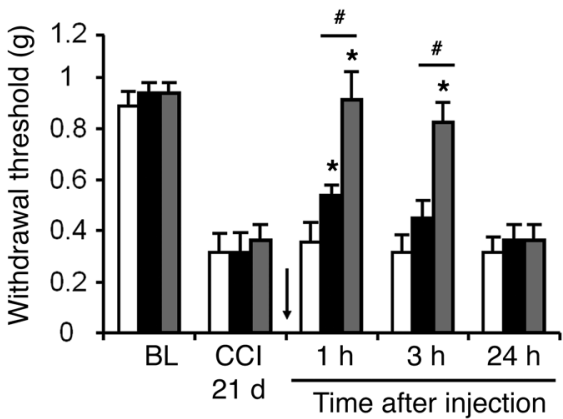

C Vehicle

BMSCs, i.t.; control IgG, $4 \mu \mathrm{g}$

BMSCs, i.t.; TGF- $\beta$ Ab, $4 \mu \mathrm{g}$

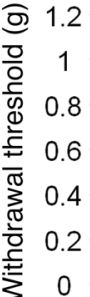

口 BMSCs, i.t.; IL-10 Ab, 4 ug

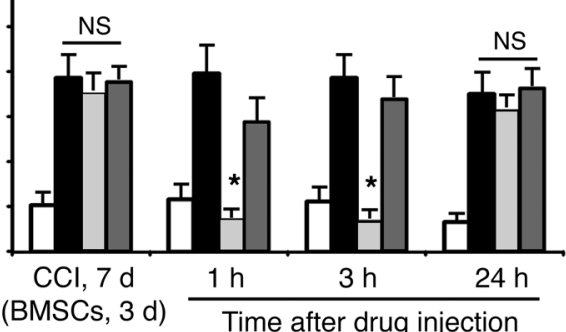

Figure 5. BMSCs release TGF- $\beta 1$ to inhibit neuropathic pain in CCI mice. (A) ELISA analysis showing TCF- $\beta 1$ and IL-10 release in BMSC culture medium and the effects of TNF $(10 \mathrm{ng} / \mathrm{ml}, 60 \mathrm{~min})$ and LPS $(100 \mathrm{ng} / \mathrm{ml}, 60 \mathrm{~min})$ on the release. ${ }^{*} P<0.05$, compared with the respective control group; $n=8$ separate cultures from different mice. (B) ELISA analysis showing increased TGF- $\beta 1$ release in CSF 8 days after CCI and 4 days after i.t. delivery of $2.5 \times 10^{5}$ BMSCs. ${ }^{*} P<0.05$, compared with naive and vehicle-treated cells; ${ }^{*} P<0.05 ; n=4$ mice/group. (C) Reversal of BMSC-induced inhibition of mechanical allodynia by TGF- $\beta 1-$ neutralizing Abs ( $4 \mu$ g, i.t.), but not by IL-10-neutralizing Abs ( $4 \mu$ g, i.t.) or control IgG ( $4 \mu g$, i.t.). ${ }^{*} P<0.05$, compared with the control IgG group; $n=5$ mice/group. (D) Reduction of TCF- $\beta 1$ release and expression in BMSCs following Tgfb1 siRNA treatment (1 $\mu$ g/ml for $18 \mathrm{~h}$ ). Both baseline release and evoked release by TNF (10 ng/ml, $1 \mathrm{~h}$ ) were measured. ${ }^{*} P<0.05$, compared with nontargeting siRNA; $P<0.05 ; n=4$ separate cultures from different mice. (E) Antiallodynic effect of BMSCs ( $2.5 \times 10^{5}$ cells) was compromised by pretreatment of BMSCs with Tgfb1 siRNA (1 $\mu$ g/ml for $\left.18 \mathrm{~h}\right)$, but not with nontargeting control siRNA. Arrow indicates the time of the BMSC injection. ${ }^{*} P<0.05$, compared with nontargeting siRNA control. $n=5$ mice/group. (F and $\left.\mathbf{G}\right)$ Dose-dependent reversal of mechanical allodynia by i.t. TCF- $\beta 1$ at 5 and 21 days after CCI. Arrows in $\mathbf{F}$ and $\mathbf{G}$ indicate the time of the BMSC injection. ${ }^{*} P<0.05$, compared with the vehicle group; ${ }^{*} P<0.05 ; n=5$ mice/group. (H) TCF- $\beta$ R1 inhibitor SB431542 (100 pmol, i.t.) completely blocked the antiallodynic effect of TGF- $\beta 1$ (10 ng, i.t.). Arrow indicates the time of i.t. injection given 19 days after $C C I .{ }^{*} P<0.05 ; n=4-5$ mice/group. Statistical significance was determined by 1-way ANOVA (A, B, and D), 2-way ANOVA, followed by Bonferroni's post-hoc test (C, E, and F-H), or Student's $t$ test (E and $\mathbf{H})$. All data are expressed as the mean \pm SEM. NT, nontargeting.

mechanical allodynia and heat hyperalgesia. TGF- $\beta 1$ delivered i.t. was highly potent at inhibiting neuropathic pain; both mechanical allodynia (Figure 5F) and thermal hyperalgesia (Supplemental Figure 5A) 5 days after CCI were dose-dependently inhibited by very low doses of TGF- $\beta 1$ ( $2 \mathrm{ng}=16 \mathrm{pmol}, 10 \mathrm{ng}=78 \mathrm{pmol})$. This reversal recovered after 24 hours (Figure $5 \mathrm{~F}$ and Supplemental Figure $5 \mathrm{~A}$ ). We also examined the effects of TGF- $\beta 1$ on established neuropathic pain. TGF- $\beta 1$ effectively inhibited late-phase neuropathic pain on day 21 after CCI (Figure 5G and Supplemental Figure 5B). We next tested whether TGF- $\beta$ receptor 1 (TGF- $\beta$ R1), the major receptor for canonical signaling of TGF- $\beta 1$ (32), would be responsible for the antiallodynic effects of TGF- $\beta 1$. Injection of SB431542 (100 pmol, i.t.), a potent and selective inhibitor of TGF- $\beta$ R1 (33), eliminated the antiallodynic and antihyperalgesic effects of TGF- $\beta 1$ (10 ng) in CCI mice (Figure 5H and Supplemental Figure 5C).

TGF- $\beta 1$ modulates excitatory synaptic transmission in spinal cord neurons after CCI via TGF- $\beta R 1$. Spinal cord synaptic plasticity (central sensitization) plays an essential role in driving neuro- 
$\mathbf{A}$

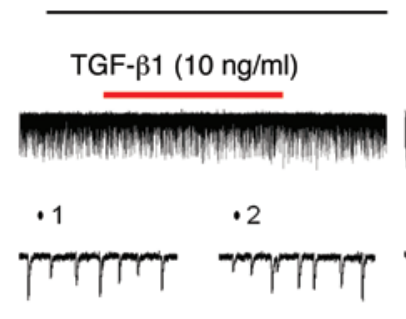

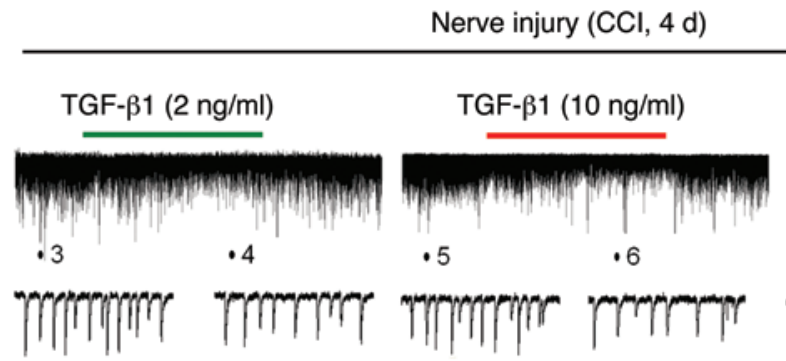

Nerve injury $(\mathrm{CCl}, 4 \mathrm{~d})$

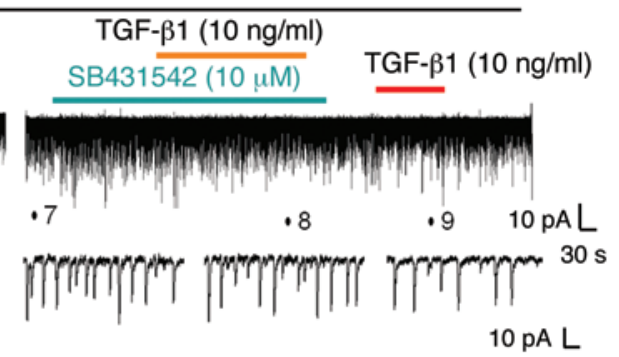

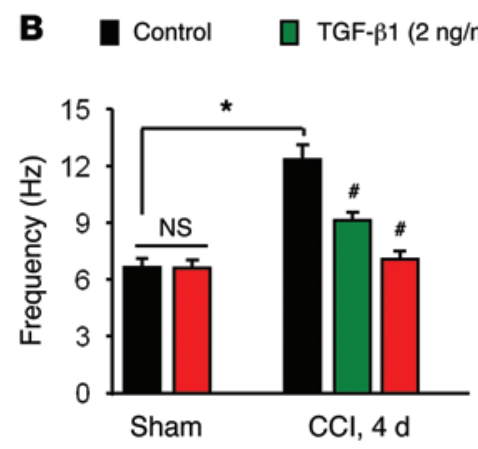
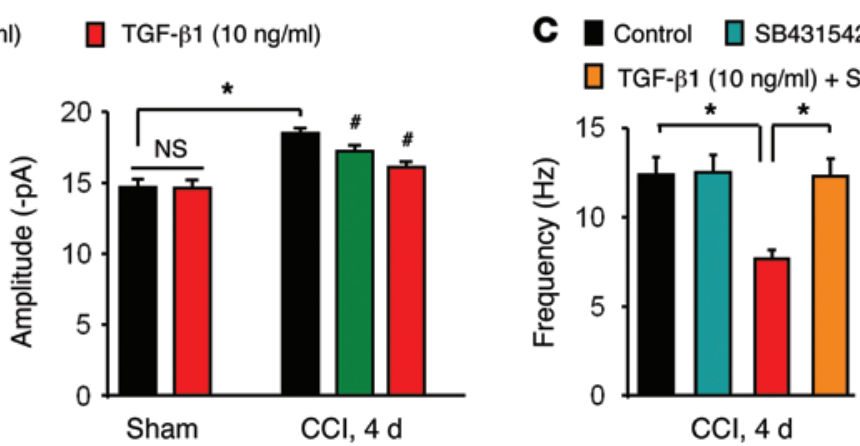

$0.1 \mathrm{~s}$

Figure 6. Exogenous TGF- $\beta 1$ rapidly suppresses CCl-induced enhancement of excitatory synaptic transmission in lamina llo neurons of spinal cord slices via the TGF-ק1R. (A) Traces showing sEPSCs in lamina llo neurons of spinal cord slices. (B) Frequency and amplitude of sEPSCs. CCI (day 4) induced profound increases in sEPSC frequency and amplitude, which were suppressed by TCF- $\beta 1$ ( 2 or $10 \mathrm{ng} / \mathrm{ml}$ ). Note that TCF- $\beta 1$ had no effect on the frequency or amplitude of sEPSCs in sham control spinal cord. ${ }^{*} P<0.05$, compared with sham surgery; ${ }^{\#} P<0.05$ compared with the control group; $n=5$ neurons/ group. (C) The TCF- $\beta 1 R$ antagonist SB431542 blocked TCF- $\beta 1$-induced inhibition of sEPSC frequency and amplitude. ${ }^{*} P<0.05 ; n=5$ neurons/group. Statistical significance was determined by 1-way ANOVA, followed by Bonferroni's post-hoc test. All data are expressed as the mean \pm SEM.

pathic pain $(34,35)$. We prepared spinal cord slices from sham and CCI mice $(4 \mathrm{~d})$ for patch-clamp recordings in lamina IIo neurons. These interneurons form a pain circuit by receiving input from C-fiber afferents and sending output to lamina I projection neurons (36). CCI resulted in significant increases in the frequency and amplitude of spontaneous excitatory postsynaptic currents (sEPSCs) in IIo neurons (Figure 6, A and B). The EPSCs are mediated by AMPA glutamate receptors, and their increases in lamina IIo neurons may drive pathological pain $(37,38)$. Superfusion of spinal cord slices with TGF- $\beta 1$ (2 or $10 \mathrm{ng} / \mathrm{ml}$ ) dose-dependently and rapidly (with 2 minutes) blocked the CCI-induced increases in sEPSC frequency and amplitude (Figure 6, A and B). Moreover, this blockade of TGF- $\beta 1$ (10 ng/ml) signaling was abrogated by the TGF- $\beta R 1$ antagonist SB431542 (10 $\mu \mathrm{M}$, Figure 6, A and C). For comparison, superfusion of TGF- $\beta 1$ (10 ng/ml) had no effect on sEPSC frequency or amplitude in sham mice (Figure 6, A and B), suggesting that TGF- $\beta 1$ selectively modulates nerve injuryinduced synaptic plasticity, but not basal synaptic transmission.

TGF- $\beta 1$ modulates neuronal excitability in DRG neurons after $C C I$ via $T G F-\beta R 1$. Neuropathic pain is also driven by hyperexcitability of primary sensory neurons $(39,40)$. We next tested the effects of TGF- $\beta 1$ on the firing frequency of evoked action potentials in small-sized DRG neurons using whole-mount DRG preparations from sham and CCI mice (41). Compared with sham surgery, CCI resulted in a profound increase in spike frequency in small-sized DRG neurons (Figure 7, A and B). TGF- $\beta 1$ rapidly (2-5 minutes) and dose dependently suppressed this increase. Further, SB431542 $(10 \mu \mathrm{M})$ reversed this effect, confirming an essential involvement of TGF- $\beta 1 \mathrm{R} 1$ in TGF- $\beta 1$ signaling (Figure 7, A and B).
BMSCs delivered i.t. target injured lumbar DRGs via the CXCL12/ CXCR 4 axis. To determine the destination of i.t.-implanted BMSCs in mice following nerve injury, we labeled BMSCs with the fluorescent dye Dil and collected DRGs and spinal cord tissues 3 days after i.t. injection of Dil-labeled BMSCs (i.e., 7 days after CCI). Interestingly, we found many labeled Dil BMSCs in the injured lumbar DRGs (L4-L6 DRGs that send axons to the sciatic nerve) 7 days after CCI (Figure 8A). In contrast, only a few Dil BMSCs were detected in contralateral DRGs (L2-L6, Figure 8A) or ipsilateral L2-L3 DRGs (Figure 8A). Dil-labeled BMSCs were also found at the borders of the white matter in the L4-L6 spinal cord, especially in the ipsilateral dorsal horn (Supplemental Figure 6A).

Chemokines play an important role in chronic pain via regulation of leukocyte trafficking, glial activation, and neuronal activity (42-45). Interestingly, CXCL12 (also named SDF-1) and its receptor CXCR4 were not only involved in neuropathic pain (46), but were also implicated in BMSC trafficking (47). CCI caused a marked increase in CXCL12 levels in L4-L6 DRGs compared with those in sham control mice (Figure 8B). Furthermore, a Transwell migration assay showed that CXCL12 induced the migration of BMSCs in vitro, and the CXCR4 antagonist AMD3100 blocked the migration of these BMSCs (Figure 8C and Supplemental Figure 7).

To define the role of the CXCL12/CXCR4 axis in regulating the migration of BMSCs in vivo, we treated BMSCs with Cxcr4 siRNA or nontargeting control siRNA $(1 \mu \mathrm{g} / \mathrm{ml}, 18$ hours $)$ and observed an $85 \%$ reduction in Cxcr4 mRNA levels after siRNA treatment (Figure 8D). Notably, BMSC-induced inhibition of mechanical allodynia was gradually (5-7 days) compromised after i.t. injection of siRNA-treated BMSCs (Figure 8E). To check the effect of 
A

Sham

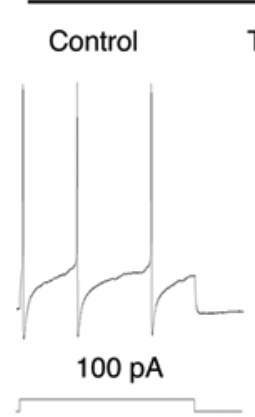

TGF- $\beta 1(10 \mathrm{ng} / \mathrm{ml})$

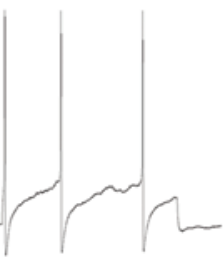

$10 \mathrm{mV}$
$100 \mathrm{~ms}$
B

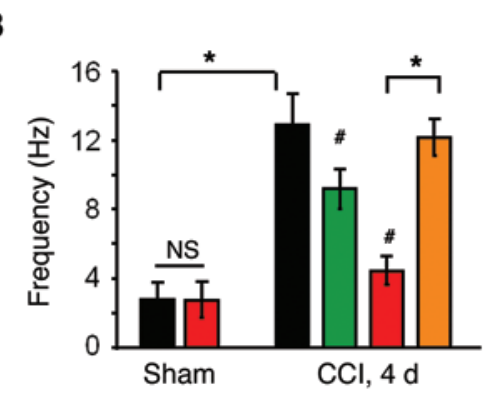

Nerve injury $(\mathrm{CCl}, 4 \mathrm{~d})$
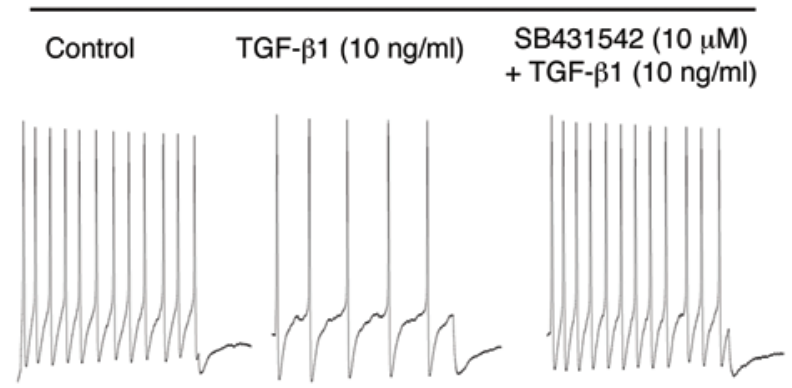

Figure 7. Exogenous TGF- $\beta 1$ blocks CCl-induced increases in action potential frequency in wholemount DRGs via the TGF- $\beta 1 R$. (A) Traces of evoked action potentials in small-sized neurons of whole-mount DRGs from sham and $\mathrm{CCI}$ mice. (B) Frequency of action potentials and effects of $\mathrm{CCl}$, TCF- $\beta 1$, and SB431542. Note that the $\mathrm{CCl}$-induced increase in action potential frequency was suppressed by TGF- $\beta 1$ ( 2 or 10 $\mathrm{ng} / \mathrm{ml})$, and this suppression was abrogated by SB431542. ${ }^{*} P<0.05$; ${ }^{\#} P<0.05$, compared with the $\mathrm{CCl}$ control. Statistical significance was determined by 1-way ANOVA, followed by Bonferroni's post-hoc test; $n=5$ neurons/group. All data are expressed as the mean \pm SEM.
Cxcr4 knockdown on BMSC trafficking, we collected ipsilateral L4-L6 DRGs from Cxcr4 siRNA- and control siRNA-treated mice 7 days after the BMSC injection. Strikingly, the number of BMSCs migrating to injured DRGs was substantially reduced in the siRNA-treated group compared with that in the control group (22.6 \pm 5.4 vs. $280.8 \pm 54.3$ per ganglion; Student's $t$ test $P<0.05 ; n=5$ mice/group) (Figure 8, F and G).

Long-term survival of BMSCs in DRGs from CCI mice following i.t. injection. To define the destination and fate of i.t.-implanted BMSCs, we collected ipsilateral L5 DRGs from CCI mice 3-84 days after their i.t. injection. The number of Dil-labeled BMSCs reached its peak on day 3 and then gradually declined (Figure 9, A and B). Notably, Dil-labeled BMSCs were detected in ipsilateral L5 DRGs, even 56 days after implantation (Figure 9, A and B), suggesting longterm survival of BMSCs in DRGs. Double-immunostaining results showed that the majority of Dil-labeled BMSCs still expressed CD90 at 28 days after CCI (Figure 9C). The BMSCs were primarily localized at the border of DRGs, and we found no evidence of genesis of neurons $\left(\mathrm{NeuN}^{+}\right)$, satellite glial cells $\left(\mathrm{GFAP}^{+}\right)$, or monocytes $\left(\mathrm{IBA1}^{+}\right.$) from the implanted BMSCs (Supplemental Figure 8, A and B). Of interest, confocal images showed Dil uptake by neurons near the BMSCs, suggesting an interaction (molecule exchange) between the infiltrated BMSCs and neurons (Supplemental Figure 8). However, the number of labeled BMSCs was dramatically reduced on day 70, and only a few BMSCs were detected on day 84 (Figure 9B). Thus, the potential health risk of i.t.-implanted BMSCs (e.g., conversion into tumor cells) should be very low.

Early or late i.t. delivery of BMSCs produces long-term relief of neuropathic pain after SNI. Finally, we tested the analgesic efficacy of BMSCs in a more persistent neuropathic pain model that was induced by SNI. In this model, mechanical allodynia is rapidly induced within 1 day and is maintained for several months in rodents (48). Injection of BMSCs $\left(2.5 \times 10^{5}\right.$ cells $)$ via the i.t. route 4 days after SNI produced a rapid ( $<1$ day) and long-lasting $(>42$ days) inhibition of SNI-induced mechanical allodynia (reduction in the paw withdrawal threshold; Figure 10A). This antiallodynic effect was more robust when mechanical allodynia was assessed by the frequency (percentage) of response to a single, low-threshold von Frey hair (0.16 grams-force [gf]; Figure 10B). BMSCs $\left(2.5 \times 10^{5}\right.$ cells $)$ delivered i.t. 3 weeks after SNI also significantly reversed late-phase mechanical allodynia for more than 5 weeks (Figure 10, C and D), although the analgesic efficacy in the SNI model was not as great as that in the CCI model (Figure 1 ). In this model, TGF- $\beta 1$ was still responsible for BMSC-induced analgesia, since the BMSC-induced antiallodynic effect was reversed by TGF- $\beta 1 \mathrm{Ab}$, given 17 days after BMSC injection (i.e., 21 days after SNI; Figure 10E).

\section{Discussion}

A number of studies reported pain relief with BMSC administration in rodent pain models following systemic or local injection, but the analgesic effects are variable (Supplemental Table 1). Percutaneous injection of autologous BM concentrate cells also caused long-term relief of lumbar discogenic pain in a pilot trial in humans (49). However, long-term analgesic effects were not fully evaluated in these animal studies (Supplemental Table 1), except via the i.v. route $(9,10)$. Our findings demonstrate that a single i.t. injection of low numbers of BMSCs $\left(1.0 \times 10^{5}-2.5 \times 10^{5}\right.$ cells $)$, either at an early time point (4 days after CCI or SNI) or at later time points (14-21 days after CCI or SNI), produced long-term relief (>4-5 weeks) from nerve injury-induced neuropathic pain symptoms, as revealed by mechanical allodynia or/and heat hyperalgesia in mice. This treatment also suppressed CCI-induced ongoing pain, reduced neuroinflammation in DRGs and spinal cord, and protected DRG neuron axons from nerve injury.

It remains unclear from previous studies how BMSCs inhibit pain. In this study, we have provided several lines of evidence to demonstrate that the analgesic effects of BMSCs are mediated by 
A
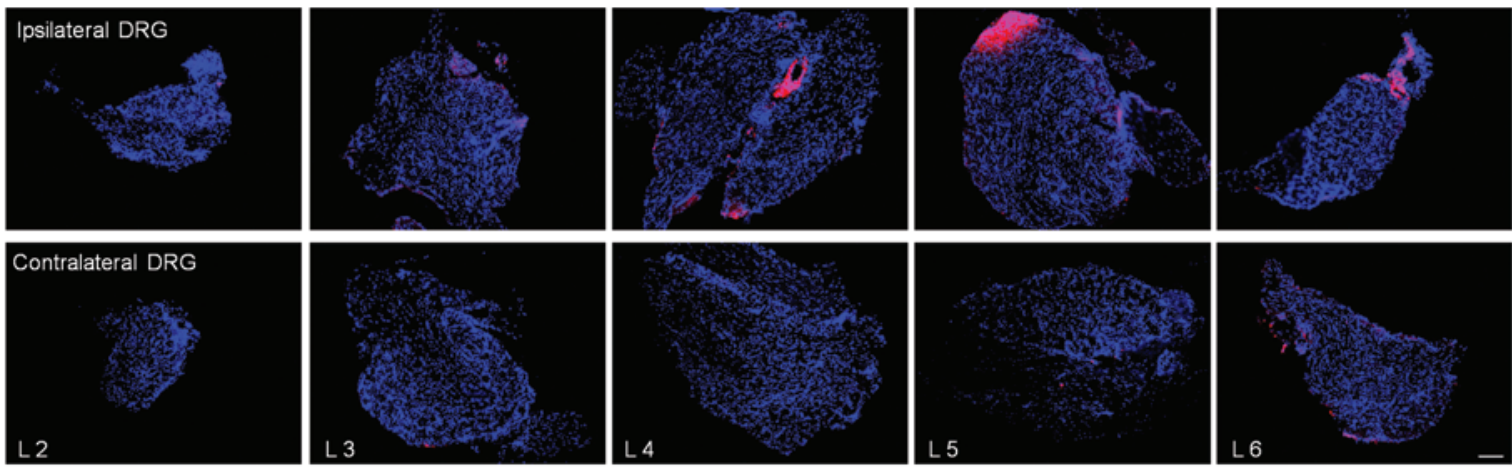

B

$\square$ Contralateral

C
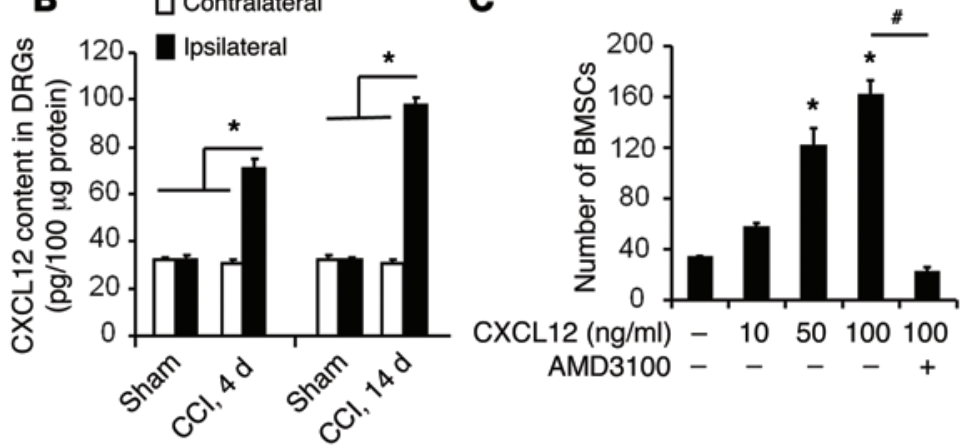

CXCL12 (ng/ml) - $10 \quad 50100100$ AMD3100
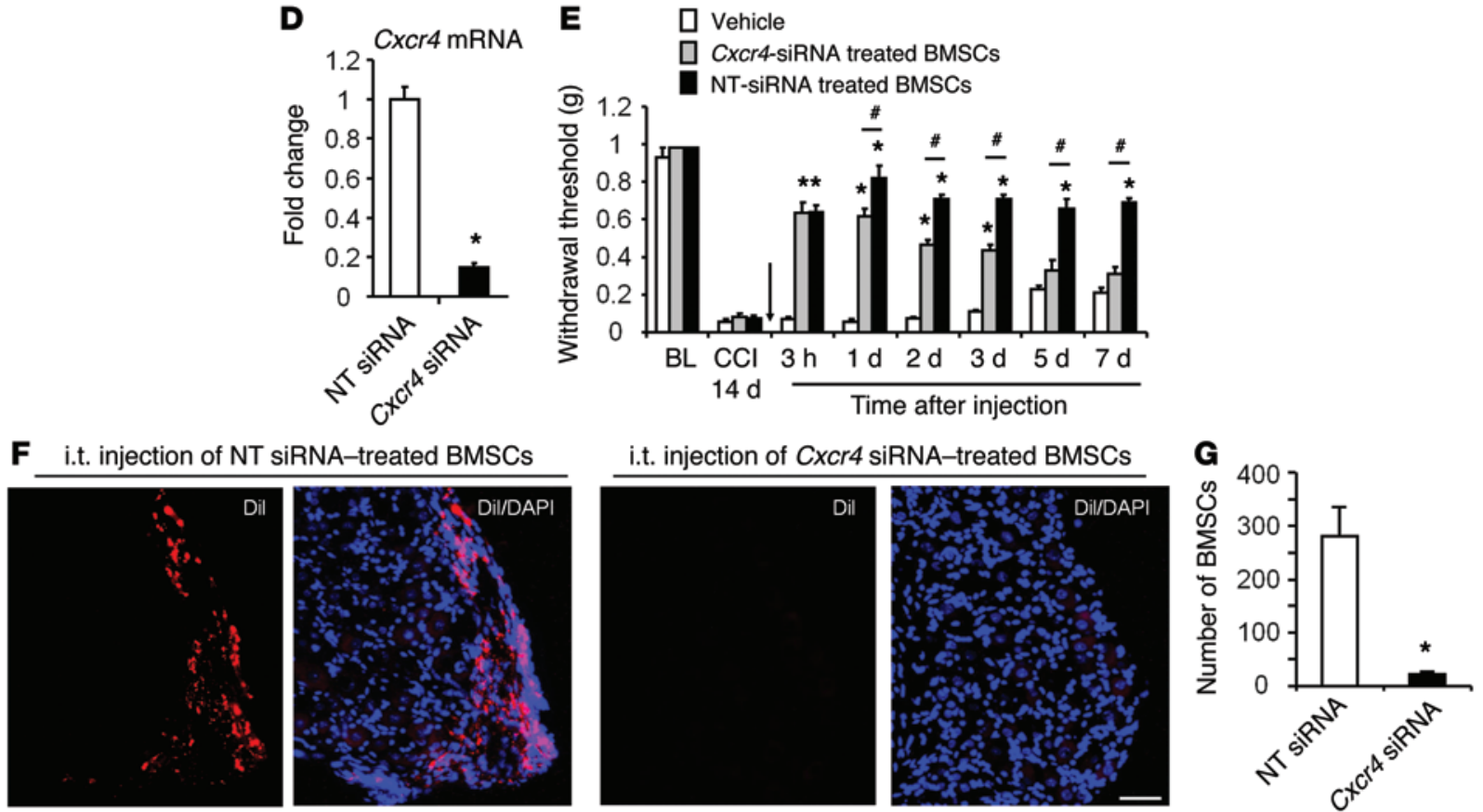

Figure 8. CXCL12/CXCR4 axis controls BMSC migration to lumbar DRGs and mediates the antiallodynic effect of BMSCs in CCI mice. (A) Selective targeting of i.t.-injected BMSCs (CM-Dil labeled) to ipsilateral L4-L6 DRGs 3 days after i.t. injection (day 7 after CCI). Scale bar: $100 \mu \mathrm{m}$. Note that there was only limited migration of BMSCs to contralateral DRGs. (B) ELISA analysis showing CXCL12 expression in contralateral and ipsilateral L4-L6 DRGs on days 4 and 14 after CCI. ${ }^{*} P<0.05 ; n=4$ mice/group. (C) Chemotaxis (Transwell invasion) assay showing the migration of BMSCs in response to CXCL12 (0-100 ng/ml) and the inhibitory effect of the CXCR4 antagonist AMD3100 (5 mg/ml, $30 \mathrm{~min}) .{ }^{*} P<0.05$, compared with the control group (no treatment); ${ }^{*} P<0.05$; $n=4$ wells from separate cultures. (D) Reduction of $C x c r 4$ mRNA levels in BMSCs treated with $C x c r 4$ siRNA $(1 \mu \mathrm{g} / \mathrm{ml}$ for $18 \mathrm{~h})$. ${ }^{*} P<0.05 ; n=3$ separate cultures. (E) Antiallodynic effect of i.t. BMSCs $\left(2.5 \times 10^{5}\right.$ cells) was compromised by pretreatment of BMSCs with $C x c r 4$ siRNA, but not with nontargeting control siRNA. Arrow indicates BMSC injection on day 14 after $C \mathrm{CI} .{ }^{*} P<0.05$, compared with the vehicle group; ${ }^{*} P<0.05 ; n=5$ mice/group. (F) Migration of CM-Dil-labeled BMSCs to ipsilateral L5 DRGs 7 days after i.t. injection (day 21 after CCI). Note that this migration was blocked by CxCr4 siRNA. Scale bar: $50 \mu \mathrm{m}$. (G) Number of CM-Dil-labeled BMSCs in ipsilateral L4-L6 DRGs after the treatment shown in F. ${ }^{*} P<0.05 ; n=5$ mice/group. Statistical significance was determined by 1-way ANOVA, followed by Bonferroni's post-hoc test (B and C), 2-way, repeated-measures ANOVA, followed by Bonferroni's post-hoc test $(\mathbf{E})$, or Student's $t$ test ( $\mathbf{D}$ and $\mathbf{G})$. All data are expressed as the mean \pm SEM. 

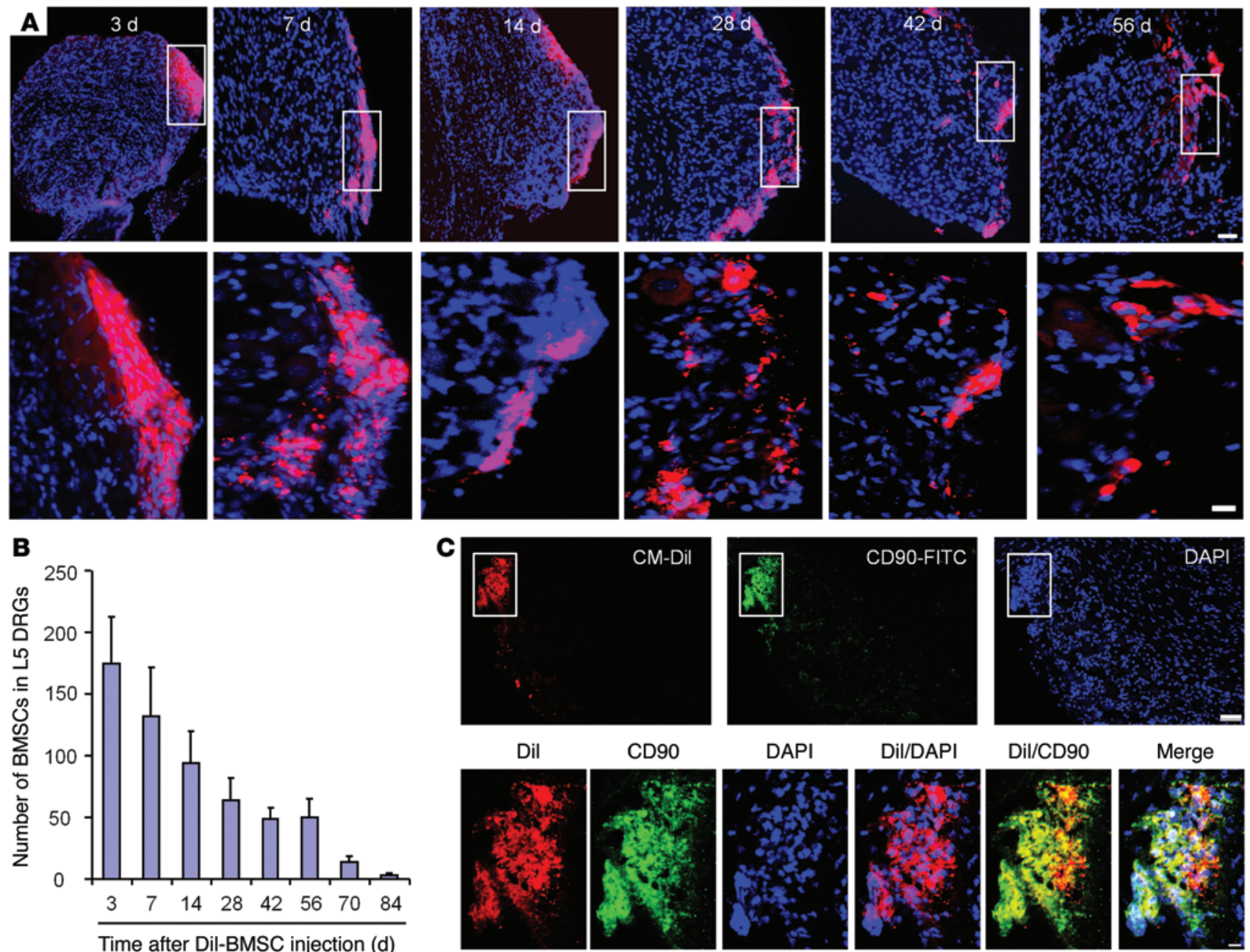

Figure 9. Long-term survival of CM-Dil-labeled BMSCs in ipsilateral L5 DRGs following i.t. injection in CCI mice. (A) Localization of CM-Dil-labeled BMSCs in ipsilateral L5 DRGs 3-56 days after i.t. injection. Scale bars: $50 \mu \mathrm{m}$ (top row) and $10 \mu \mathrm{m}$ (bottom row). Bottom panels are enlarged images of the top panels. Note that BMSCs were mainly localized at DRG borders. (B) Number of CM-Dil-labeled BMSCs in L5 DRGs 3-84 days after i.t. BMSC injection, given 4 days after CCI. $n=4$ mice/group. (C) CM-Dil-labeled BMSCs expressed the stem cell marker CD90 in L5 DRGs 28 days after BMSC injection. Bottom panels are high-magnification merged images of the top panels. Scale bars: $50 \mu \mathrm{m}$ (upper) and $10 \mu \mathrm{m}$ (lower).

TGF- $\beta 1$ secretion. First, TGF- $\beta 1$ levels in CSF dramatically increased after i.t. implantation of BMSCs. There was also a sustained increase in TGF- $\beta 1$ expression in lumbar DRGs in CCI mice after i.t. BMSC administration. Second, the antiallodynic and antihyperalgesic effects of BMSCs were blocked by TGF- $\beta 1$, but not IL-10, neutralization. Third, the analgesic effects of BMSCs pretreated with Tgfb1 siRNA were compromised. Fourth, i.t. administration of exogenous TGF- $\beta 1$ potently inhibited neuropathic pain after CCI. In contrast, IL-10 release from BMSCs was very low and did not contribute to BMSC-induced pain relief. How does TGF- $\beta 1$ reduce neuropathic pain? As a major antiinflammatory cytokine, TGF- $\beta 1$ inhibits the activation and proliferation of microglia and astrocytes and reduces the expression of proinflammatory cytokines in neuropathic pain conditions $(30,50)$. As expected, i.t. BMSCs inhibited CCI-induced neuroinflammation in DRGs and spinal cord (Figure 4). We also identified an unconventional role of TGF- $\beta 1$ by showing rapid action of TGF- $\beta 1$ on neurons. It is striking that within minutes, TGF- $\beta 1$ normalized nerve injury-induced spinal cord synaptic plasticity and DRG neuronal hyperexcitability (Figures 6 and 7). Such fast action of neuromodulation cannot be explained by canonical TGF- $\beta 1$ signaling, which requires gene transcription. Further studies are needed to characterize this rapid and unconventional signaling of TGF- $\beta 1$ in neurons. However, we should not rule out that i.t. BMSCs may attenuate chronic pain via TGF- $\beta 1$-independent mechanisms.

Another important finding of this study is that of selective targeting of i.t. administered BMSCs to the injured lumbar DRGs after CCI. The long-term survival of BMSCs in DRGs is correlated with sustained pain relief by BMSCs. Intriguingly, the migration of CXCR4-expressing BMSCs to the injured DRGs was triggered by CXCL12 upregulation in these DRGs. Thus, knockdown of Cxcr4 in BMSCs not only blocked the migration of BMSCs to DRGs, but also shortened the duration of BMSC-induced analgesia. This is consistent with a previous report that BMSCs from Cxcr4-KO mice exhibited reduced migration to the brain subjected to permanent middle cerebral artery occlusion and impaired functional recovery (47). It is noteworthy that we observed many BMSCs in lumbar DRGs even 8 weeks after injection of BMSCs into CCI mice. Although these cells exhibited persistent expression of CD90, they 

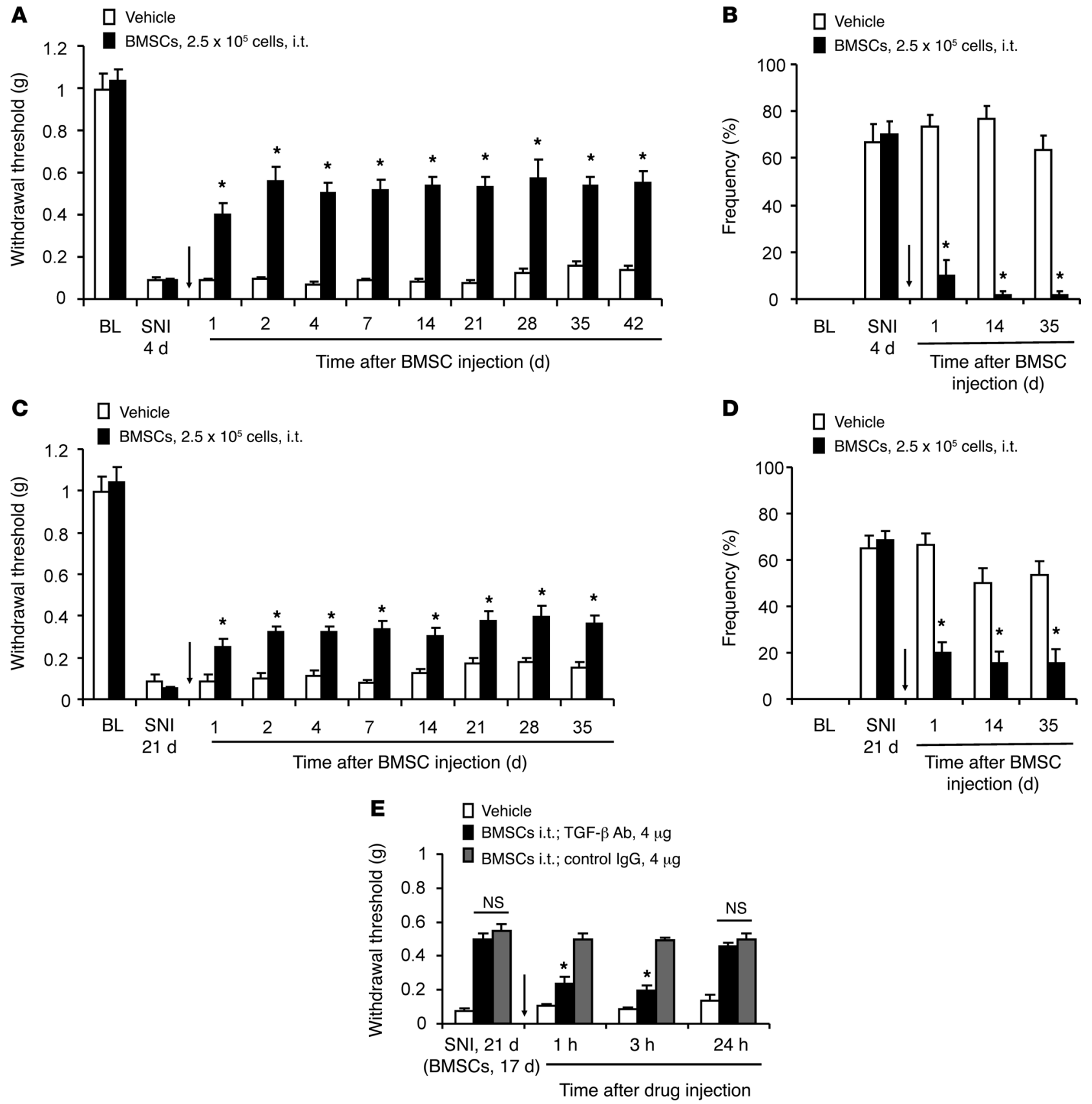

Figure 10. Prolonged inhibition of SNI-induced mechanical allodynia by i.t. BMSCs in the early or late phase and its reversal by TGF- $\beta 1$ Abs. (A and B) Long-term inhibition of mechanical allodynia by early treatment with BMSCs $\left(2.5 \times 10^{5}\right.$ cells) via i.t. injection, given 4 days after SNI. Mechanical allodynia was tested using the paw withdrawal threshold (A) and by the frequency of response (percentage of response) to a single $0.16 \mathrm{gf}$ filament (10 times) (B). ${ }^{*} P<0.05$, compared with vehicle (PBS); $n=6$ mice/group. (C and $\mathbf{D}$ ) Sustained inhibition of mechanical allodynia by late treatment with i.t. BMSCs (2.5 $\times 10^{5}$ cells), given 21 days after SNI. ${ }^{*} P<0.05$, compared with vehicle; $n=6$ mice/group. (E) Reversal of BMSC-induced inhibition of mechanical allodynia by i.t. TCF- $\beta 1$-neutralizing Abs $(4 \mu \mathrm{g})$, given 3 weeks after SNI. Arrows in A-E indicate the time of injection. ${ }^{*} P<0.05$, compared with the IgG control group; $n=5$ mice/group. All data are expressed as the mean \pm SEM. Statistical significance was determined by 2-way ANOVA, followed by Bonferroni's post-hoc test.

were primarily localized at the border of DRGs and spinal cord and did not migrate deep into these tissues; nor were they converted into neurons, satellite cells, or monocytes. These BMSCs were eliminated from DRGs within 3 months, thus posing a low risk of carcinogenesis. Our data also support the notion that the beneficial effects of BMSC transplantation in animal models are more likely due to paracrine actions than to the effective integration and differentiation of BMSCs within the host tissue $(7,28,51)$.
The potential of treating chronic pain with stem cells via spinal implantation has been explored. An early study showed that neuropathic pain limits the application of intraspinal neural stem cell grafts, since the implanted stem cells in the injured spinal cord differentiated into astrocytes to elicit allodynia (52). However, direct spinal implantation of forebrain GABAergic neuroprogenitor cells has been shown to alleviate neuropathic pain by integrating into the spinal cord pain circuit (53). Although this study is promising, 
spinal implantation of stem cells is very invasive and may be more suitable for some patients with spinal cord injury. BMSCs were also injected directly into the lesion site via the intraganglionic or intrajoint route $(9,12)$ or administered i.v. $(9,10)$. However, the additional trauma from intramedullary transplantation can further compromise injured tissue and impede clinical translation. BMSCs administered i.v. in different animal models revealed that transplanted BMSCs are mostly confined to the lung $(28,54)$. Furthermore, BMSCs delivered via the systemic route only survive for a few days $(28,55)$. As a comparison, there are several advantages of using the i.t. route for BMSC delivery. First, it is minimally invasive compared with direct injection into the injured tissues. Second, the small i.t. space allows for injection of small amounts $\left(10^{5}\right.$ cells) of TGF- $\beta 1$ to reach therapeutic concentrations. Third, the i.t. space is protected by the blood-brain/spinal cord barrier; there is only minimal infiltration of immune cells, which could be suppressed by TGF- $\beta 1$ secreted from BMSCs. Most important, the implanted BMSCs migrate to the injured lumbar DRGs and/ or spinal cord tissues through the CXCL12/CXCR4 axis, leading to long-term survival in these tissues and sustained analgesic and neuroprotective effects.

In summary, our findings demonstrate that i.t.-injected BMSCs could produce sustained neuropathic pain relief in mice via TGF- $\beta 1$ secretion. Our data support the notion that grafted stem cells can serve as "in vivo drug stores" (7) to synthesize and release paracrine factors for therapeutic benefits. Although i.t. infusion of TGF- $\beta 1$ via a pump could be an option, direct systemic administration of TGF- $\beta 1$ or its agonist may cause side effects such as diabetes (56). In contrast to the limited success of currently available drugs in treating devastating neuropathic pain, BMSCs offer distinct advantages for the efficacious treatment of chronic pain. These cells can be further engineered to produce and secret more TGF- $\beta$ and other neuroprotective and antiinflammatory factors. Enhancing the function of the CXCL12/CXCR4 axis during BMSCs delivery may further improve the analgesic efficacy of the implanted BMSCs.

\section{Methods}

Animals and surgery. Adult male CD1 mice (25-32 g) were purchased from Charles River Laboratories and used for behavioral studies and primary cultures of BMSCs. Neuropathic pain was produced by CCI of the sciatic nerve (19) and by SNI (48). To produce the CCI model, animals were anesthetized with isoflurane, and the left sciatic nerve was exposed and 3 ligatures (7-0 prolene) placed around the nerve proximal to the trifurcation with 1 millimeter between each ligature. The ligatures were loosely tied until a short flick of the ipsilateral hind limb was observed (15). Animals in the sham group underwent surgery identical to that described in ref. 48, but without nerve ligation. For the SNI surgery, the mice were anesthetized with isoflurane, and a 5.0 silk tight ligation of the tibial and common peroneal nerves was performed, followed by transection and removal of a 3- to 5-mm portion of the nerve. However, the third peripheral branch of the sciatic nerve, the sural nerve, was left intact, and any contact with or stretching of this nerve was carefully avoided. The number of mice used and the experiments performed are summarized in Supplemental Table 2.

Drugs and administration. TGF- $\beta 1$, TGF- $\beta$-neutralizing $\mathrm{Ab}$, TNF, and CXCL12/SDF-1 $\alpha$ were purchased from R\&D Systems; LPS from Sigma-Aldrich; AMD3100 (CXCR4 antagonist) and SB431542
(TGF- $31 \mathrm{R}$ inhibitor) from Selleckchem; IL-10-neutralizing Ab from BioLegend; and normal Rabbit IgG from Santa Cruz Biotechnology Inc. For i.t. injection, a spinal cord puncture was made with a 30 -gauge needle between L5 and L6 levels to deliver reagents $(10 \mu \mathrm{l})$ or cells ( 1 or $2.5 \times 10^{5}$ cells in $10 \mu \mathrm{l}$ PBS) to the CSF (57). Before injection, BMSCs were washed 3 times with $0.01 \mathrm{M}$ PBS, centrifuged for 5 minutes at $1,000 \mathrm{~g}$, and then resuspended in PBS. In some cases, BMSCs were incubated with Vybrant CM-Dil Cell-Labeling Solution (Molecular Probes, Life Technologies) for 20 minutes at $37^{\circ} \mathrm{C}$. Cells were then washed 3 times with PBS and resuspended.

Tgfb1 siRNA (catalog J-040652-06), Cxcr4 siRNA (catalog J-060184-06), and nontargeting siRNA (catalog D-0018100-01-20) were purchased from Thermo Scientific Dharmacon. SiRNA was dissolved in RNase-free water at $1 \mu \mathrm{g} / \mu \mathrm{l}$ as a stock solution and mixed with the transfection reagent polyethyleneimine (PEI) (Fermentas, Life Technologies) and normal saline before use. Specifically, $1 \mu \mathrm{g}$ siRNA was dissolved in $3.3 \mu \mathrm{l}$ PEI and $66 \mu \mathrm{l}$ normal saline (58).

BMSC cultures and flow cytometric analysis. Primary cultures of BMSCs were obtained from donor mice under aseptic conditions. The mice were sacrificed with isoflurane, and the tibiae and femurs were cut off with scissors. A syringe fitted with a 20-gauge needle was inserted into the shaft of the bone, and BM was flushed out with modified Eagle's medium. The cells were cultured in $75-\mathrm{cm}^{2}$ flasks or 6-well culture plates. The properties of the expanded cells were assessed by flow cytometry using FITC-conjugated mAbs against CD45 (1:400; eBioscience) and CD90 (1:400; eBioscience) and analyzed using FlowJo software. Additional details are provided in the Supplemental Methods.

ELISA. Mouse TGF- $\beta 1$, IL-10, and CXCL12/SDF-1 $\alpha$ ELISA kits were purchased from $R \& D$ Systems. ELISA was performed using BMSC culture medium or cell lysate and CSF and DRG tissue. Cultured cells and DRGs were homogenized in a lysis buffer containing protease and phosphatase inhibitors. CSF was collected from the cisterna magna 4 or 28 days after i.t. injection of BMSCs and diluted at 1:10 with $1 \times$ Calibrator Diluent RD5-53 (Quantikine ELISA; R\&D Systems). For each assay, $50 \mu \mathrm{g}$ protein, $50 \mu \mathrm{l}$ culture medium, or $5 \mu \mathrm{l} \mathrm{CSF}$ was used, and ELISA was performed according to the manufacturer's instructions. The standard curve was included in each experiment.

IHC. Animals were deeply anesthetized with isoflurane and perfused through the ascending aorta with PBS, followed by $4 \%$ paraformaldehyde. After perfusion, spinal cord segments and DRGs were removed and postfixed in the same fixative overnight. Spinal cord and DRG sections were cut in a cryostat and processed for immunofluorescence. The sections were incubated overnight at $4^{\circ} \mathrm{C}$ with the following primary Abs: mouse GFAP (1:1,000; EMD Millipore); rabbit IBA-1 (1:1,000; Wako Chemicals USA Inc.); rabbit CGRP (1:1,000; Abcam); rabbit ATF-3 (1:1,000; Santa Cruz Biotechnology Inc.); mouse NeuN (1:1,000; EMD Millipore); and rat CD90(1:200; BD Biosciences). Fluorescein-labeled isolectin B4 $(10 \mu \mathrm{g} / \mathrm{ml}$; Sigma-Aldrich) was used to label IB4-binding cells. In some cases, DAPI (Vector Laboratories) was used to stain cell nuclei. The stained sections were examined under a Nikon fluorescence microscope or a Zeiss confocal microscope. The intensity of fluorescence was analyzed using ImageJ software (NIH). Further details are provided in the Supplemental Methods.

Trafficking of BMSCs to DRGs. To examine the distribution of transplanted CM-Dil-labeled BMSCs following i.t. injection, lumbar spinal cord segments and L1-L6 DRGs were collected. For quantita- 
tive analysis of engrafted cells in DRGs, 10 sections $(12 \mu \mathrm{m})$ from each DRG were examined for the labeled BMSCs.

qPCR. Spinal dorsal horn tissues of L4-L5 segments were rapidly isolated. Total RNA was extracted using the RNeasy Plus Mini kit (QIAGEN), and 0.5-1 $\mu$ g RNA was reverse transcribed. Specific primers, including GAPDH control, were designed using IDT SciTools Real-Time PCR software (Integrated DNA Technologies Inc). We performed gene-specific mRNA analyses using the MiniOpticon Real-Time PCR system (BioRad). qPCR amplifications contained the same amount of reverse transcription (RT) product, including $7.5 \mu \mathrm{l}$ $2 \times$ iQ SYBR Green Supermix (BioRad) and 100-300 nM forward and reverse primers in a final volume of $15 \mu \mathrm{l}$. The primer sequences were described in our previous study (38). Primer efficiency was obtained from the standard curve and integrated for the calculation of relative gene expression, which was based on the real-time PCR threshold values of different transcripts and groups.

Transwell migration assay. The migratory ability of BMSCs was determined using Transwell plates $(6.5 \mathrm{~mm}$ in diameter with $8-\mu \mathrm{m}$ pore filters; Corning Costar). In brief, $5 \times 10^{4}$ cells in $100 \mu$ l serum-free medium were added into the upper well, and $600 \mu \mathrm{l} \mathrm{CXCL12-contain-}$ ing medium was placed in the lower well of a Transwell plate. Following incubation for 5 hours $\left(37^{\circ} \mathrm{C}, 100 \%\right.$ humidity, $5 \% \mathrm{CO}_{2}$ in air), the number of cells that had migrated to the lower side of the filter was counted under a light microscope at $\times 200$ magnification (data are presented as the average number of migratory BMSCs in 5 randomly selected fields). Each experiment was performed in triplicate, and the data were averaged for statistical analysis.

Patch-clamp recordings in spinal cord slices. As we previously reported (38), a portion of the lumbar spinal cord (L4-L5) was removed from 4- to 7-week-old mice under urethane anesthesia (1.5$2.0 \mathrm{~g} / \mathrm{kg}$, i.p.) and was kept in preoxygenated ice-cold Krebs solution. Transverse slices (400-600 $\mu \mathrm{m})$ were cut on a vibrating microslicer. The slices were perfused with Krebs solution $(8-10 \mathrm{ml} / \mathrm{min})$ that was saturated with $95 \% \mathrm{O}_{2}$ and $5 \% \mathrm{CO}_{2}$ at $36^{\circ} \mathrm{C}$ for at least 1 to 3 hours prior to the experiment. The Krebs solution contained $117 \mathrm{mM} \mathrm{NaCl}$, $3.6 \mathrm{mM} \mathrm{KCl}, 2.5 \mathrm{mM} \mathrm{CaCl}_{2}, 1.2 \mathrm{mM} \mathrm{MgCl}_{2}, 1.2 \mathrm{mM} \mathrm{NaH}_{2} \mathrm{PO}_{4}, 25 \mathrm{mM}$ $\mathrm{NaHCO}_{3}$, and $11 \mathrm{mM}$ glucose. Whole-cell patch-clamp recordings were made from lamina IIo neurons in voltage-clamp mode (38). Patch pipettes were fabricated from thin-walled borosilicate glass-capillary tubing (1.5 mm outer diameter; World Precision Instruments). After establishing the whole-cell configuration, neurons were held at their holding potentials at $-70 \mathrm{mV}$ for recording of sEPSCs. The resistance of a typical patch pipette is $5-10 \mathrm{M} \Omega$. The internal solution contained $135 \mathrm{mM}$ potassium gluconate, $5 \mathrm{mM} \mathrm{KCl}, 0.5 \mathrm{CaCl}_{2}, 2 \mathrm{mM} \mathrm{MgCl}, 5$ mM EGTA, 5 mM HEPES, and $5 \mathrm{mM}$ ATP-Mg. Membrane currents were amplified with an Axopatch 200B Amplifier (Molecular Devices) in voltage-clamp mode. Signals were filtered at $2 \mathrm{kHz}$ and digitized at 5 $\mathrm{kHz}$. Data were stored with a personal computer using pClamp 6 software and analyzed with Mini Analysis (Synaptosoft).

Whole-cell patch-clamp recordings in whole-mount DRGs. As shown in our previous study (41), L4-L5 whole-mount DRGs were carefully removed from the vertebral column and placed in cold oxygenated ACSF. The connective tissue was gently removed under a microscope, and the ganglia were digested with a mixture of $1.0 \mathrm{mg} / \mathrm{ml}$ protease and $1.6 \mathrm{mg} / \mathrm{ml}$ collagenase (Sigma-Aldrich) for 30 minutes at $37^{\circ} \mathrm{C}$. The ganglion was transferred into a holding chamber containing normal $\mathrm{Mg}^{2+}$-free artificial CSF (ACSF) with CNQX $(2 \mu \mathrm{M})$ bubbled with $95 \% \mathrm{O}_{2}$ and $5 \% \mathrm{CO}_{2}$ at room temperature. Whole-cell currentclamp recordings were performed at room temperature $\left(28^{\circ} \mathrm{C}\right)$ for the measurement of action potentials with an Axopatch 200B Amplifier (Axon Instruments) (41). The patch pipettes were pulled from borosilicate capillaries (Chase Scientific Glass Inc.). When filled with the pipette solution, the resistance of the pipettes was 4-5 M $\Omega$. The recording chamber $(300 \mu \mathrm{l})$ was continuously superfused $(3-4 \mathrm{ml} /$ min). Series resistance was compensated for (>80\%), and leak subtraction was performed. Data were low-pass filtered at $2 \mathrm{KHz}$ and sampled at $10 \mathrm{KHz}$. pClamp10 (Axon Instruments) software was used for the experiments and analysis.

Behavioral analysis. Animals were habituated to the testing environment for at least 2 days before baseline testing. The room temperature and humidity remained stable for all experiments. All mouse behaviors were tested blindly. To test mechanical sensitivity, we confined the mice in boxes placed on an elevated metal mesh floor and stimulated their hind paws with a series of von Frey hairs with logarithmically increasing stiffness (0.02-2.56 gf; Stoelting), presented perpendicularly to the central plantar surface. We determined the $50 \%$ paw withdrawal threshold by Dixon's up-down method. Mechanical allodynia after SNI was also assessed according to the frequency of response (expressed as the percentage of response) to a low-threshold von Frey hair (0.16 gf, 10 times). Thermal sensitivity was tested using a Hargreaves radiant heat apparatus (IITC Life Science), with the basal paw withdrawal latency adjusted to 9 to 12 seconds and a cutoff of 20 seconds to prevent tissue damage. A Randall-Selitto analgesiometer (Ugo Basile) was used to examine mechanical sensitivity by applying ascending pressure to a mouse tail, with a cutoff threshold of 250 gf to avoid tissue damage. A rotarod system (IITC Life Science) was used to assess motor function. Mice were tested in 3 trials separated by 10-minute intervals. During the tests, the speed of rotation was accelerated from 2 to 20 rpm over a 3-minute period. The fall latency was recorded and averaged.

To test spontaneous/ongoing pain measurement, we used a single-trial conditioning protocol for $\operatorname{CPP}(17,38)$. All mice underwent a 3-day preconditioning habituation, and animal behavior was video recorded. Analyses of the preconditioning (baseline) behavior showed no preexisting chamber preference. On the conditioning day, mice received the vehicle (saline) control paired with a randomly chosen chamber in the morning, then the appropriate treatment (clonidine, $10 \mu \mathrm{g}$, i.t.) paired with the other chamber 4 hours later. Chamber pairings were counterbalanced. On the test day, 20 hours after the afternoon pairing, mice were placed in the CPP box with access to both chambers, and their behavior was recorded for 15 minutes and analyzed for chamber preference by ANY-maze software (Stoelting).

Statistics. All data are expressed as the mean \pm SEM. The sample size for each experiment is summarized in Supplemental Table 2 and is also described in the figure legends. Behavioral data were analyzed by 2-way, repeated-measures ANOVA, followed by Bonferroni's post-hoc test. Differences between 2 groups were compared using the Student's $t$ test. One-way ANOVA was used for the statistical analyses in other tests. The criterion for statistical significance was a $P$ value of less than 0.05 .

Study approval. All the animal procedures performed in this study were approved by the Animal Care Committee of Duke University Medical Center. 


\section{Acknowledgments}

This study was supported by NIH grants R01NS67686, R01NS87988, R01NS89479, R01DE17794, and R01 DE22743

(to R.R. Ji).
Address correspondence to: Ru-Rong Ji or Gang Chen, Department of Anesthesiology, Duke University Medical Center, Durham, North Carolina 27701, USA. Phone: 919.684.9387, E-mail: ru-rong. ji@duke.edu (R.R. Ji), gang.chen@duke.edu (G. Chen).
1. Costigan M, Scholz J, Woolf CJ. Neuropathic pain: a maladaptive response of the nervous system to damage. Annu Rev Neurosci. 2009;32:1-32.

2. Farrar JT. Advances in clinical research methodology for pain clinical trials. Nat Med. 2010;16(11):1284-1293.

3. Finnerup NB, Sindrup SH, Jensen TS. The evidence for pharmacological treatment of neuropathic pain. Pain. 2010;150(3):573-581.

4. Pittenger MF, et al. Multilineage potential of adult human mesenchymal stem cells. Science. 1999;284(5411):143-147.

5. Giordano A, Galderisi U, Marino IR. From the laboratory bench to the patient's bedside: an update on clinical trials with mesenchymal stem cells. J Cell Physiol. 2007;211(1):27-35.

6. Parr AM, Tator CH, Keating A. Bone marrowderived mesenchymal stromal cells for the repair of central nervous system injury. Bone Marrow Transplant. 2007;40(7):609-619.

7. Caplan AI, Correa D. The MSC: an injury drugstore. Cell Stem Cell. 2011;9(1):11-15.

8. Jones BJ, McTaggart SJ. Immunosuppression by mesenchymal stromal cells: from culture to clinic. Exp Hematol. 2008;36(6):733-741.

9. Guo W, et al. Bone marrow stromal cells produce long-term pain relief in rat models of persistent pain. Stem Cells. 2011;29(8):1294-1303.

10. Siniscalco D, et al. Long-lasting effects of human mesenchymal stem cell systemic administration on pain-like behaviors, cellular, and biomolecular modifications in neuropathic mice. Front Integr Neurosci. 2011;5:79.

11. Siniscalco D. Transplantation of human mesenchymal stem cells in the study of neuropathic pain. Methods Mol Biol. 2010;617:337-345.

12. Musolino PL, Coronel MF, Hokfelt T, Villar MJ. Bone marrow stromal cells induce changes in pain behavior after sciatic nerve constriction. Neurosci Lett. 2007;418(1):97-101.

13. van Buul GM, et al. Mesenchymal stem cells reduce pain but not degenerative changes in a mono-iodoacetate rat model of osteoarthritis. JOrthop Res. 2014;32(9):1167-1174.

14. Naruse K, et al. Transplantation of bone marrow-derived mononuclear cells improves mechanical hyperalgesia, cold allodynia and nerve function in diabetic neuropathy. PLoS One. 2011;6(11):e27458.

15. Bennett GJ, Xie YK. A peripheral mononeuropathy in rat that produces disorders of pain sensation like those seen in man. Pain. 1988;33(1):87-107.

16. Mogil JS. Animal models of pain: progress and challenges. Nat Rev Neurosci. 2009;10(4):283-294.

17. King T, et al. Unmasking the tonic-aversive state in neuropathic pain. Nat Neurosci. 2009;12(11):1364-1366.

18. Tsujino $\mathrm{H}$, et al. Activating transcription factor 3 (ATF3) induction by axotomy in sensory and motoneurons: A novel neuronal marker of nerve injury. Mol Cell Neurosci. 2000;15(2):170-182.
19. Bennett DL, et al. A distinct subgroup of small DRG cells express GDNF receptor components and GDNF is protective for these neurons after nerve injury. J Neurosci. 1998;18(8):3059-3072.

20. Gardell LR, et al. Multiple actions of systemic artemin in experimental neuropathy. Nat Med. 2003;9(11):1383-1389.

21. Ji RR, Xu ZZ, Gao YJ. Emerging targets in neuroinflammation-driven chronic pain. Nat Rev Drug Discov. 2014;13(7):533-548.

22. Ellis A, Bennett DL. Neuroinflammation and the generation of neuropathic pain. Br J Anaesth. 2013;111(1):26-37.

23. Ji RR, Berta T, Nedergaard M. Glia and pain: is chronic pain a gliopathy? Pain. 2013; 154(suppl 1):S10-S28.

24. Calvo M, Dawes JM, Bennett DL. The role of the immune system in the generation of neuropathic pain. Lancet Neurol. 2012;11(7):629-642.

25. Coull JA, et al. BDNF from microglia causes the shift in neuronal anion gradient underlying neuropathic pain. Nature. 2005;438(7070):1017-1021.

26. Milligan ED, Watkins LR. Pathological and protective roles of glia in chronic pain. Nat Rev Neurosci. 2009;10(1):23-36.

27. McMahon SB, Malcangio M. Current challenges in glia-pain biology. Neuron. 2009;64(1):46-54.

28. Nemeth K, et al. Bone marrow stromal cells use TGF- $\beta$ to suppress allergic responses in a mouse model of ragweed-induced asthma. Proc Natl Acad Sci U S A. 2010;107(12):5652-5657.

29. Nemeth K, et al. Bone marrow stromal cells attenuate sepsis via prostaglandin E(2)-dependent reprogramming of host macrophages to increase their interleukin-10 production. Nat Med. 2009;15(1):42-49.

30. Echeverry S, Shi XQ, Haw A, Liu H, Zhang ZW, Zhang J. Transforming growth factor- $\beta 1$ impairs neuropathic pain through pleiotropic effects. $\mathrm{Mol}$ Pain. 2009;5:16.

31. Milligan ED, et al. Intrathecal polymer-based interleukin-10 gene delivery for neuropathic pain. Neuron Glia Biol. 2006;2(4):293-308.

32. Schmierer B, Hill CS. TGFbeta-SMAD signal transduction: molecular specificity and functional flexibility. Nat Rev Mol Cell Biol. 2007;8(12):970-982.

33. Inman GJ, et al. SB-431542 is a potent and specific inhibitor of transforming growth factor- $\beta$ superfamily type I activin receptor-like kinase (ALK) receptors ALK4, ALK5, and ALK7. Mol. Pharmacol. 2002;62(1):65-74.

34. Woolf CJ, Salter MW. Neuronal plasticity: increasing the gain in pain. Science. 2000;288(5472):1765-1769.

35. Luo C, Kuner T, Kuner R. Synaptic plasticity in pathological pain. Trends Neurosci. 2014;37(6):343-355.

36. Todd AJ. Neuronal circuitry for pain processing in the dorsal horn. Nat Rev Neurosci.
2010;11(12):823-836

37. Gangadharan V, et al. Peripheral calciumpermeable AMPA receptors regulate chronic inflammatory pain in mice. J Clin Invest. 2011;121(4):1608-1623.

38. Xu ZZ, et al. Neuroprotectin/Protectin D1 protects neuropathic pain in mice after nerve trauma. Ann Neurol. 2013;74(3):490-495.

39. Campbell JN, Meyer RA. Mechanisms of neuropathic pain. Neuron. 2006;52(1):77-92.

40. Hucho T, Levine JD. Signaling pathways in sensitization: toward a nociceptor cell biology. Neuron. 2007;55(3):365-376.

41. Lee JH, et al. A monoclonal antibody that targets a NaV1.7 channel voltage sensor for pain and itch relief. Cell. 2014;157(6):1393-1404.

42. Zhang J, Shi XQ, Echeverry S, Mogil JS, De Koninck Y, Rivest S. Expression of CCR2 in both resident and bone marrow-derived microglia plays a critical role in neuropathic pain. J Neurosci. 2007;27(45):12396-12406.

43. Clark AK, Staniland AA, Malcangio M. Fractalkine/CX3CR1 signalling in chronic pain and inflammation. Curr Pharm Biotechnol. 2011;12(10):1707-1714.

44. Gao YJ, Ji RR. Chemokines, neuronal-glial interactions, and central processing of neuropathic pain. Pharmacol Ther. 2010;126(1):56-68.

45. Milligan ED, et al. Evidence that exogenous and endogenous fractalkine can induce spinal nociceptive facilitation in rats. Eur J Neurosci. 2004;20(9):2294-2302.

46. Bhangoo SK, et al. CXCR4 chemokine receptor signaling mediates pain hypersensitivity in association with antiretroviral toxic neuropathy. Brain Behav Immun. 2007;21(5):581-591.

47. Shichinohe H, Kuroda S, Yano S, Hida K, Iwasaki Y. Role of SDF-1/CXCR4 system in survival and migration of bone marrow stromal cells after transplantation into mice cerebral infarct. Brain Res. 2007;1183:138-147.

48. Decosterd I, Woolf CJ. Spared nerve injury: an animal model of persistent peripheral neuropathic pain. Pain. 2000;87(2):149-158.

49. Pettine KA, Murphy MB, Suzuki RK, Sand TT. Percutaneous injection of autologous bone marrow concentrate cells significantly reduces lumbar discogenic pain through 12 months. Stem Cells. 2015;33(1):146-156

50. Chen NF, et al. TGF-beta1 attenuates spinal neuroinflammation and the excitatory amino acid system in rats with neuropathic pain. JPain. 2013;14(12):1671-1685

51. Cantinieaux D, et al. Conditioned medium from bone marrow-derived mesenchymal stem cells improves recovery after spinal cord injury in rats: an original strategy to avoid cell transplantation. PLoS One. 2013;8(8):e69515.

52. Hofstetter CP, et al. Allodynia limits the usefulness of intraspinal neural stem cell grafts; directed differentiation improves outcome. Nat 


\section{RESEARCH ARTICLE}

Neurosci. 2005;8(3):346-353.

53. Braz JM, et al. Forebrain GABAergic neuron precursors integrate into adult spinal cord and reduce injury-induced neuropathic pain. Neuron. 2012;74(4):663-675.

54. Lee RH, et al. Intravenous hMSCs improve myocardial infarction in mice because cells embolized in lung are activated to secrete the anti-inflammatory protein TSG-6. Cell Stem Cell. 2009;5(1):54-63.

55. Karp JM, Leng Teo GS. Mesenchymal stem cell homing: the devil is in the details. Cell Stem Cell. 2009;4(3):206-216.

56. Yan J, et al. Obesity- and aging-induced excess of central transforming growth factor- $\beta$ potentiates diabetic development via an RNA stress
The Journal of Clinical Investigation

response. Nat Med. 2014;20(9):1001-1008. 57. Hylden JL, Wilcox GL. Intrathecal morphine in mice: a new technique. Eur J Pharmacol. 1980;67(2):313-316.

58. Kawasaki Y, et al. Distinct roles of matrix metalloproteases in the early- and late-phase development of neuropathic pain. Nat Med. 2008;14(3):331-336. 